\title{
Fourier Transforms of Pulses Containing Exponential Leading and Trailing Profiles
}

S.I. Warshaw

July 15, 2001

U.S. Department of Energy

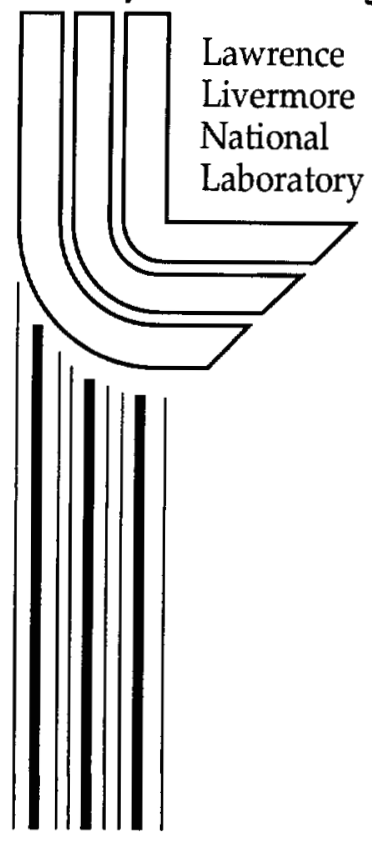




\section{DISCLAIMER}

This document was prepared as an account of work sponsored by an agency of the United States Government. Neither the United States Government nor the University of California nor any of their employees, makes any warranty, express or implied, or assumes any legal liability or responsibility for the accuracy, completeness, or usefulness of any information, apparatus, product, or process disclosed, or represents that its use would not infringe privately owned rights. Reference herein to any specific commercial product, process, or service by trade name, trademark, manufacturer, or otherwise, does not necessarily constitute or imply its endorsement, recommendation, or favoring by the United States Government or the University of California. The views and opinions of authors expressed herein do not necessarily state or reflect those of the United States Government or the University of California, and shall not be used for advertising or product endorsement purposes.

This work was performed under the auspices of the U. S. Department of Energy by the University of California, Lawrence Livermore National Laboratory under Contract No. W-7405-Eng-48.

This report has been reproduced directly from the best available copy.

Available electronically at http://www.doc.gov/bridge

Available for a processing fee to U.S. Department of Energy

And its contractors in paper from

U.S. Department of Energy

Office of Scientific and Technical Information

P.O. Box 62

Oak Ridge, TN 37831-0062

Telephone: (865) 576-8401

Facsimile: (865) 576-5728

E-mail: reports@adonis.osti.gov

Available for the sale to the public from

U.S. Department of Commerce

National Technical Information Service

5285 Port Royal Road

Springfield, VA 22161

Telephone: (800) 553-6847

Facsimile: (703) 605-6900

E-mail: orders@ntis.fedworld.gov

Online ordering: http://www.ntis.gov/ordering.htm

OR

Lawrence Livermore National Laboratory

Technical Information Department's Digital Library

http://www.llnl.gov/tid/Library.html 
UCRL-ID- $x x x x x x x x x$

Fourier Transforms of Pulses Containing

Exponential Leading and Trailing Profiles

Stephen I. Warshaw

15 July 2001

N Division

Physics and Advanced Technologies Directorate Lawrence Livermore National Laboratory Livermore, California 94551 
Table of Contents

1. Introduction 3

2. Evaluation of Three Integrals 3

3. The Inverse Biexponential Pulse Shape 6

4. Sum of Two Inverse Biexponential Functions 10

5. A Fractal Family of Inverse Biexponential Functions 15

6. Bipolar Forms of Inverse Biexponential Functions 21

7. Extensions to Boxcar and N Wave Functions 25

8. Epilogue: Formulas and Algorithms 29

$\begin{array}{ll}\text { References } & 31\end{array}$

Appendix: Complex Gamma Function Subroutine 33 
Fourier Transforms of Pulses Containing

Exponential Leading and Trailing Profiles

Stephen I. Warshaw

N Division, Physics and Advanced Technologies Directorate Lawrence Livermore National Laboratory, Livermore CA 94551

\section{Introduction}

In this monograph we discuss a class of pulse shapes that have exponential rise and fall profiles, and evaluate their Fourier transforms. Such pulses can be used as models for time-varying processes that produce an initial exponential rise and end with the exponential decay of a specified physical quantity. Unipolar examples of such processes include the voltage record of an increasingly rapid charge followed by a damped discharge of a capacitor bank, and the amplitude of an electromagnetic pulse produced by a nuclear explosion. (See, e.g., Northrop (1996), Radasky (1988) and references they cite.) Bipolar examples include acoustic $\mathrm{N}$ waves propagating for long distances in the atmosphere that have resulted from explosions in the air, and sonic booms generated by supersonic aircraft. These bipolar pulses have leading and trailing edges that appear to be exponential in character. (See, e.g., Pierce (1981), Whitham (1974), Lighthill (1956).)

To the author's knowledge the Fourier transforms of such pulses are not generally well-known or tabulated in Fourier transform compendia, and it is the purpose of this monograph to derive and present these transforms. These Fourier transforms are related to a definite integral of a ratio of exponential functions, whose evaluation we carry out in considerable detail. From this result we derive the Fourier transforms of other related functions. In all Figures showing plots of calculated curves, the actual numbers used for the function parameter values and dependent variables are arbitrary and non-dimensional, and are not identified with any particular physical phenomenon or model.

\section{Evaluation of Three Integrals}

We establish the following first integral result by the residue theorem, thus following a similar derivation carried out by Faulkner (1988):

$$
\int_{-\infty}^{\infty} \frac{e^{\lambda x} d x}{1+e^{x}}=\frac{\pi}{\sin \pi \lambda}
$$


where $\mathrm{x}$ is a real variable and $\lambda$ is a complex constant such that $0<\operatorname{Re} \lambda<1$. We evaluate the contour integral of the complex function $\mathrm{e}^{\lambda \mathrm{z}} /\left(1+\mathrm{e}^{\mathrm{z}}\right)$ counter-clockwise along a closed curve in the complex plane consisting of two segments: a semicircle of radius $\mathrm{R}$ in the upper half plane with center at the origin, and a line segment along the real axis from $x=-R$ to $x=+R$ connecting the ends of the semicircle. This contour integral is then equal to $2 \pi i$ times the sum of the residues at the poles of the integrand lying within the contour. The poles are located at points $z_{0}$ where the integrand denominator vanishes, that is, at values $z=z_{0}$ for which $e^{z_{0}}=-1$. Thus $z_{0}=(2 n+1) \pi i$ and the poles lie entirely on the imaginary axis. Now, if the limit of $\left(z-z_{0}\right) f(z)$ as $z \rightarrow z_{0}$ exists and is finite, then the poles of $f(z)$ at $z_{0}$ are simple, and the residues at these poles are precisely this limiting result. It further follows that if the function $f(z)$ is a ratio of two analytic functions $p(z) / q(z)$, then the residue of $f(z)$ at the poles $z_{0}$ of $f(z)$ (the zeros of $q(z)$ ) is given by $p\left(z_{0}\right) / q^{\prime}\left(z_{0}\right)$. (See, e.g., Churchill (1961) or Nehari (1961).) This is indeed so for the function $\mathrm{e}^{\lambda z} /\left(1+\mathrm{e}^{z}\right)$; the residues at $\mathrm{z}_{0}$ are therefore $\mathrm{e}^{(\lambda-1) z_{0}}$. The contour evaluation is then, in full,

$$
\oint \frac{\mathrm{e}^{\lambda z} \mathrm{dz}}{1+\mathrm{e}^{\mathrm{z}}}=\int_{-\mathrm{R}}^{\mathrm{R}} \frac{\mathrm{e}^{\lambda x} \mathrm{dx}}{1+\mathrm{e}^{\mathrm{x}}}+\mathrm{R} \int_{0}^{\pi} \frac{\mathrm{e}^{\lambda \mathrm{z}} \mathrm{d \theta}}{1+\mathrm{e}^{\mathrm{z}}}=2 \pi \mathrm{i} \sum_{\mathrm{n}} \mathrm{e}^{(\lambda-1)(2 \mathrm{n}+1) \pi \mathrm{i}}
$$

where the first definite integral is along the straight segment on the real axis, the second (angular) one is along the semicircular arc (for which $z=\operatorname{Re}^{\mathrm{i} \theta}$ ), and in the summation $\mathrm{n}$ runs from 0 to the index of the highest zero lying within the contour. As the radius $\mathrm{R} \rightarrow \infty$ it is clear that $\mathrm{n} \rightarrow \infty$ also, and the limiting value of the first definite integral is the desired result.

The angular integral can be shown to vanish as $\mathrm{R} \rightarrow \infty$, because the absolute value of any line integral of an analytic function has an upper bound of $M_{c} L_{c}$, where $\mathrm{M}_{\mathrm{c}}$ is at least the maximum absolute value of the integrand on the contour, and $\mathrm{L}_{\mathrm{c}}$ is the length of the contour. Symbolically, this says that

$$
\left|\int_{c} f(z) d z\right| \leq M_{c} L_{c} \text {. }
$$

For the angular integral, we find that $M_{c}=1 /(2 \cosh (\lambda R \cos \theta))$ and $L_{c}=\pi R$, and so $\mathrm{M}_{\mathrm{c}} \mathrm{L}_{\mathrm{c}} \rightarrow 0$ as $\mathrm{R} \rightarrow \infty$. Applying this maximal criterion to the first integral also determines certain restrictive conditions on the parameter $\lambda$, because the integral should be bounded as $x \rightarrow \pm \infty$. We thus require $\operatorname{Re} \lambda>0$, for otherwise the integral would diverge as the lower limit $-R \rightarrow-\infty$. (This is a consequence of the exponent $x$ in the denominator of the integrand having $a+$ sign.) For large $R$ we have $M_{c} \rightarrow$ $\mathrm{e}^{(R e \lambda-1) \mathrm{R}}$, and so we further require that $\operatorname{Re} \lambda<1$, in order for the integral to converge as the upper limit $\mathrm{R} \rightarrow+\infty$. 
Turning now to the summation above, Faulkner astutely observed that its terms constitute a geometric series when the common term $\mathrm{e}^{(\lambda-2 n-1) \pi \mathrm{i}}=-\mathrm{e}^{\lambda \pi \mathrm{i}}$ is factored out. The term-to-term ratio of the resulting series is $r=e^{2 \lambda \pi i}$; its infinite sum is of the form $\Sigma \mathrm{r}^{\mathrm{n}}$ and thus evaluates to $1 /(1-\mathrm{r})=1 /\left(1-\mathrm{e}^{2 \lambda \pi \mathrm{i}}\right)$. The right-hand side of the contour integral equation then becomes $-2 \pi i e^{\lambda \pi i} /\left(1-e^{2 \lambda \pi i}\right)$, which easily reduces to $\pi / \sin \lambda \pi$. Q.E.D.

The next integral result we wish to establish is similar to the previous one:

$$
\int_{-\infty}^{\infty} \frac{\mathrm{e}^{\lambda x} \mathrm{dx}}{\left(1+\mathrm{e}^{\mathrm{x}}\right)^{2}}=\frac{(1-\lambda) \pi}{\sin \pi \lambda}
$$

The evaluation of this integral proceeds almost exactly as in the previous case, even with the poles of the integrand occurring at the same locations $z_{0}=(2 n+1) \pi i$ as before. However, the order of these poles is 2 , because in the limit as $z \rightarrow z_{0}$ the quantity $\left(z-z_{0}\right)^{2} e^{\lambda x} /\left(1+e^{x}\right)^{2}$ exists and is finite. For this case the residues at the poles are evaluated by carrying out the limit operation given by

$$
\lim _{z \rightarrow z_{0}} \frac{d}{d z}\left(\frac{\left(z-z_{0}\right)^{2} e^{\lambda z}}{\left(1+e^{z}\right)^{2}}\right)
$$

and are found to be $(\lambda-1) \mathrm{e}^{\lambda z_{0}}$. The subsequent evaluation then proceeds in the manner carried out for the previous case, from which the asserted result follows.

We may verify this result by noting that

$$
\int_{-\infty}^{\infty} \frac{\mathrm{e}^{\lambda x} \mathrm{dx}}{\mu+\mathrm{e}^{\mathrm{x}}}=\frac{\mu^{\lambda-1} \pi}{\sin \pi \lambda}
$$

easily follows from the first integral result. By differentiating both sides of this equation with respect to $\mu$ and subsequently setting $\mu=1$, we obtain the second integral result. This process can obviously be carried out for higher orders of the integrand denominator by repeated differentiation with respect to $\mu$, and then setting $\mu=1$ afterwards; the generic result after $\mathrm{k}$ such differentiations is

$$
\int_{-\infty}^{\infty} \frac{\mathrm{e}^{\lambda x} \mathrm{dx}}{\left(\mu+\mathrm{e}^{\mathrm{x}}\right)^{\mathrm{k}}}=\frac{(1-\lambda)(2-\lambda) \ldots .(\mathrm{k}-1-\lambda)}{(\mathrm{k}-1) !} \frac{\mu^{\lambda-\mathrm{k}} \pi}{\sin \pi \lambda} .
$$

This evaluation procedure is much more preferable and easier to use than the method of residues for the higher orders of the denominator, because the residue at 
a pole of $f(z)$ of order $k$ (where the limit of $\left(z-z_{0}\right)^{k} f(z)$ as $z \rightarrow z_{0}$ exists and is finite) is usually evaluated from the limiting value of a multiple order derivative, viz.

$$
\frac{1}{(k-1) !} \lim _{z \rightarrow z_{0}} \frac{d^{k-1}}{d z^{k-1}}\left\{\left(z-z_{0}\right)^{k} f(z)\right\}
$$

Such evaluations can be extremely tedious at high orders.

The third integral result we need is obtained by differentiating the first integral result with respect to the parameter $\lambda$ (Shaeffer (2001)). This leads to the integral evaluation

$$
\int_{-\infty}^{\infty} \frac{x \mathrm{e}^{\lambda x} \mathrm{dx}}{1+\mathrm{e}^{\mathrm{x}}}=-\frac{\pi^{2} \cos \pi \lambda}{\sin ^{2} \pi \lambda} .
$$

Other related integrals quickly follow. One such arises from using the relation $2 \mathrm{e}^{\mathrm{x}} \cosh \mathrm{x}=1+\mathrm{e}^{2 \mathrm{x}}$ in the integrand of the first integral to produce the following result:

$$
\int_{-\infty}^{\infty} \frac{e^{(2 \lambda-1) x} d x}{\cosh x}=\frac{\pi}{\sin \pi \lambda}
$$

Another is obtained by making the integration variable substitution $y=e^{x}$ in the first integral to yield

$$
\int_{0}^{\infty} \frac{y^{\lambda-1} d y}{1+y}=\frac{\pi}{\sin \lambda \pi} .
$$

This result is also derived by contour integration in Nehari.

These integral results have been previously reported by Gradshteyn and Ryzhik (1965) and Jeffrey (1995). We end this section by noting that all are related to the gamma function through the remarkable reflection formula (see, e.g. Whittaker and Watson (1927))

$$
\Gamma(x) \Gamma(1-x)=\frac{\pi}{\sin \pi x} .
$$

3. The Inverse Biexponential Pulse Shape

The simplest instance of a uniformly continuous pulse shape with exponential rise and fall profiles is the inverse biexponential function given by 


$$
f(t)=\frac{1}{e^{-\alpha\left(t-t_{0}\right)}+e^{\beta\left(t-t_{0}\right)}}
$$

where $\alpha$ and $\beta$ are positive real numbers. This function has exponential leading and trailing edge asymptotes $\mathrm{e}^{\alpha\left(t-t_{0}\right)}$ and $\mathrm{e}^{-\beta\left(t-t_{0}\right)}$ respectively. It approaches zero as $\mathrm{t}$ approaches either infinity, and peaks near $t=t_{0}$, where $t_{0}$ is the value of $t$ at which the asymptotes intersect. (In this paper we shall as a convenience regard this intersection point as the time location of the pulse for relative phase purposes, to be discussed in the next section.) The peak occurs at $\mathrm{t}_{\text {peak }}=\mathrm{t}_{0}+(\ln \alpha-\ln \beta) /(\alpha+\beta)$, and the peak value of the function is $f\left(t_{\text {peak }}\right)=1 /\left[(\beta / \alpha)^{\alpha /(\alpha+\beta)}+(\alpha / \beta)^{\beta /(\alpha+\beta)}\right]$.

The Fourier transform of this function can be written

$$
F(\omega)=\int_{-\infty}^{+\infty} f(t) e^{i \omega t} d t=e^{i \omega t_{0}} \int_{-\infty}^{+\infty} \frac{e^{i \omega \tau} d \tau}{e^{-\alpha \tau}+e^{\beta \tau}}
$$

where we assume the positive sign convention for i $\omega t$ (see, e.g., Jeffrey), and define the integration variable $\tau=\mathrm{t}-\mathrm{t}_{0}$. Further manipulation of parameters and exponents leads to

$$
F(\omega)=\frac{e^{i \omega t_{0}}}{\alpha+\beta} \int_{-\infty}^{+\infty} \frac{e^{\lambda x} d x}{1+e^{x}}=\xi e^{i \omega t_{0}} \csc ((\alpha+i \omega) \xi)
$$

where $\lambda=(\alpha+i \omega) /(\alpha+\beta), x=(\alpha+\beta) \tau, \xi=\pi /(\alpha+\beta)$, and we make use of the first basic integral result derived before. We observe that one of the main integral parameter conditions is always satisfied, viz., $\operatorname{Re} \lambda=\alpha /(\alpha+\beta)<1$. We also note that the asymptote crossover time $t_{0}$ determines the transform phase factor $\mathrm{e}^{\mathrm{i} \omega \mathrm{t}_{0}}$. The magnitude of the Fourier transform is easily evaluated and is

$$
|F(\omega)|=\frac{\xi}{\sqrt{\sin ^{2}(\xi \alpha)+\sinh ^{2}(\xi \omega)}} .
$$

Note that $F(0)=\xi / \sin (\xi \alpha)$, and that $|F(\omega)| \rightarrow 2 \xi e^{-\xi \omega}$ as $\omega \rightarrow \infty$. Thus $F(\omega)$ levels off as $\omega \rightarrow 0$ and decreases exponentially as $\omega \rightarrow \infty$.

We plot $f(t)$ in Figures 1 and 2, and the magnitude $|F(\omega)|$ in Figures 3 and 4, using, respectively, log-linear, linear-linear, $\log -\log$ and $\log$-linear axes, and with arbitrary units. The asymptotic behavior of both $\mathrm{f}(\mathrm{t})$ and $|\mathrm{F}(\omega)|$ is clearly evident in the log-linear plots. 


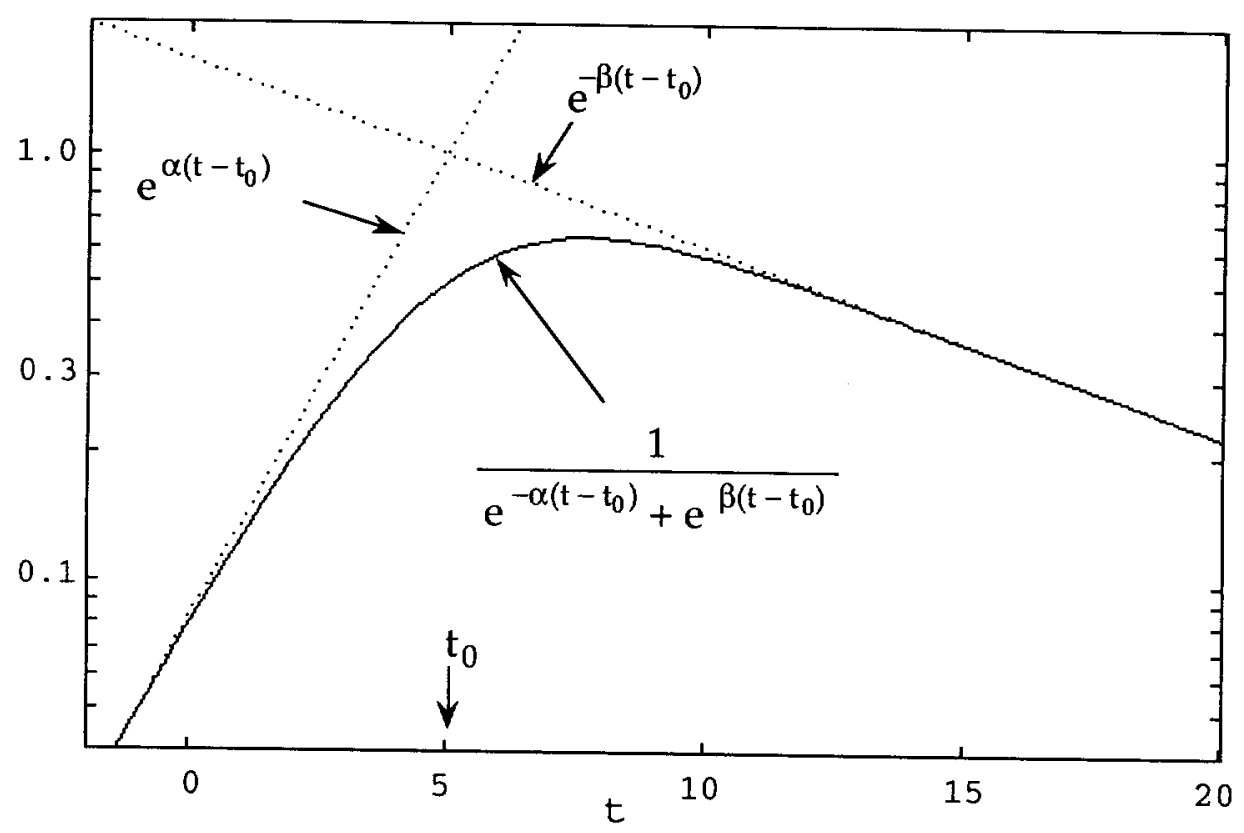

Figure 1. Semi-log plot of the inverse biexponential function $f(t)$ and its two asymptotes (shown dotted), using $\alpha=0.5, \beta=0.1$ and $t_{0}=5$.

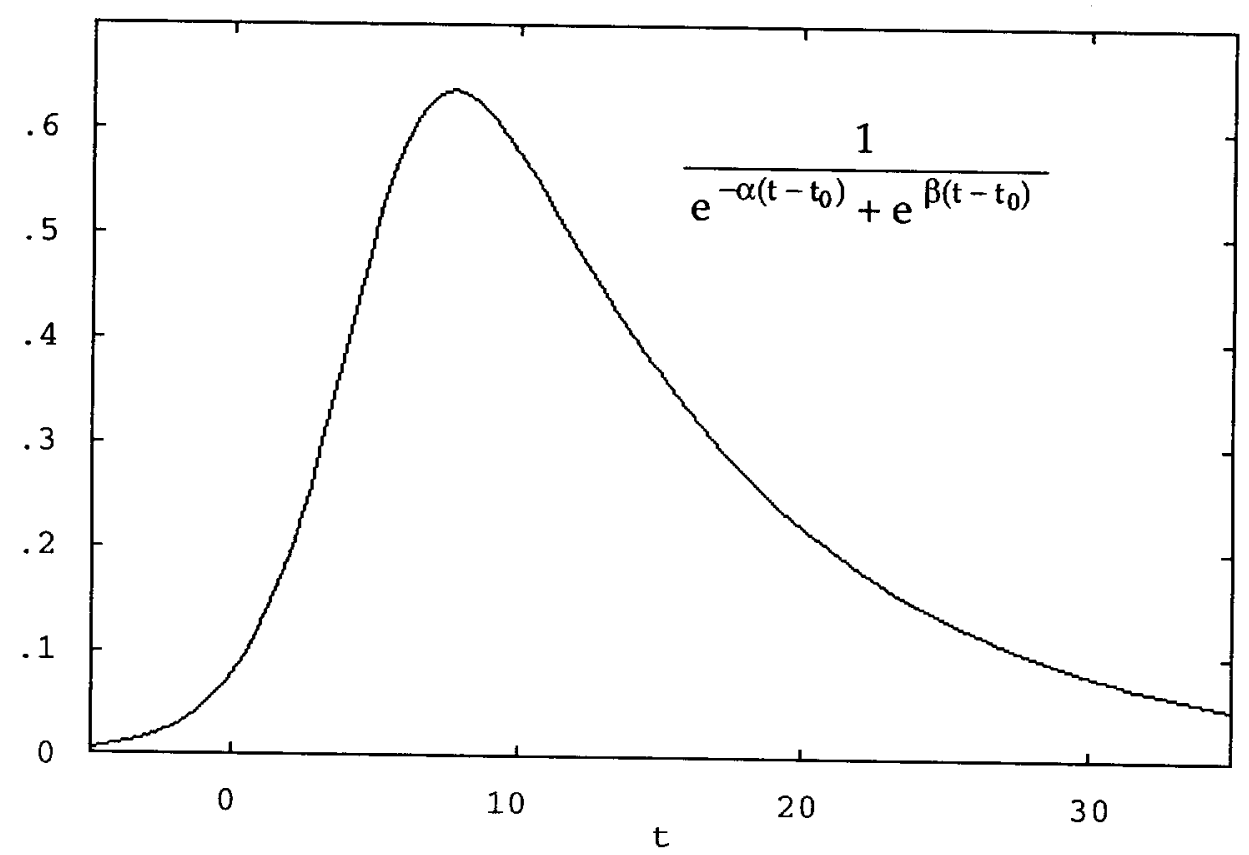

Figure 2. Linear-linear plot of the inverse biexponential function $\mathrm{f}(\mathrm{t})$ with $\alpha=0.5, \beta=0.1$ and $\mathrm{t}_{0}=5$. 


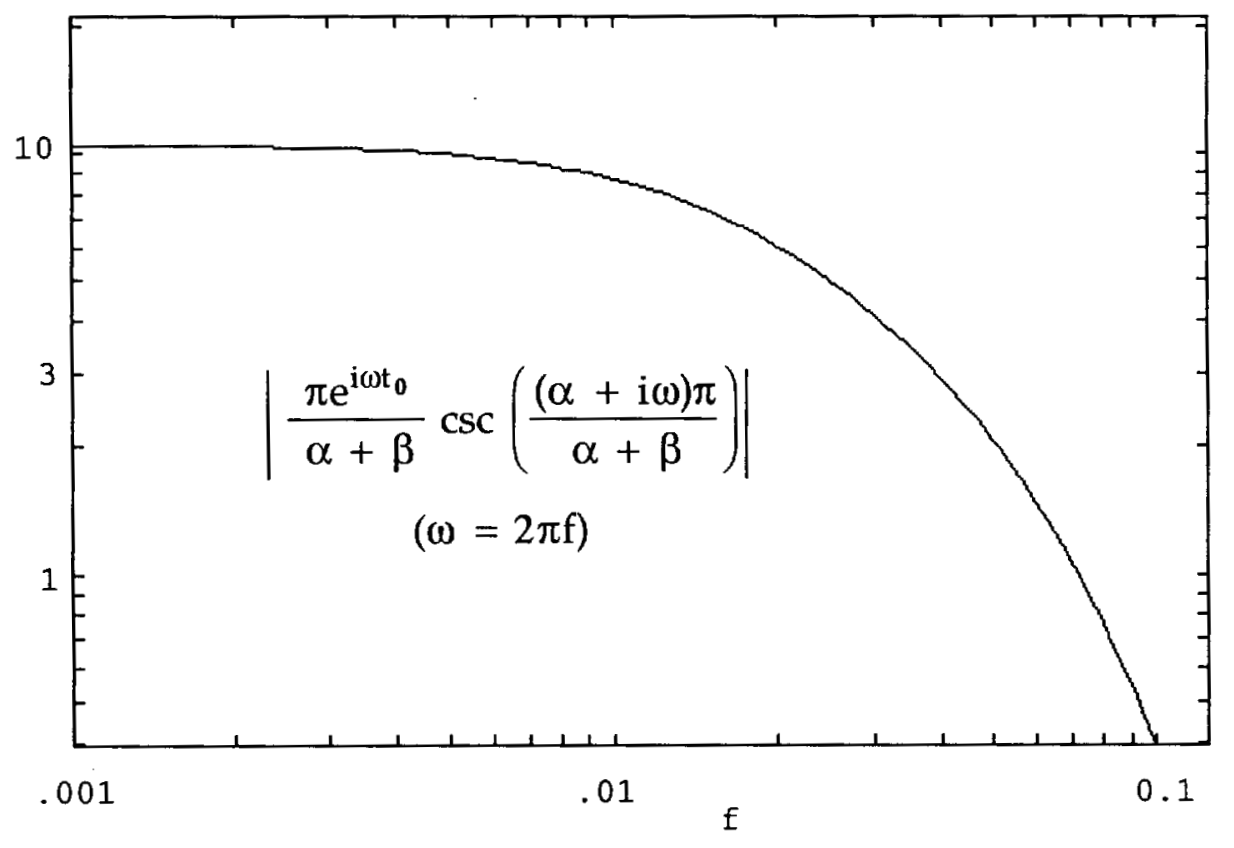

Figure 3. Log-log plot of the magnitude $|F(\omega)|$ of the Fourier transform of the inverse biexponential function, using $\alpha=0.5$ and $\beta=0.1$.

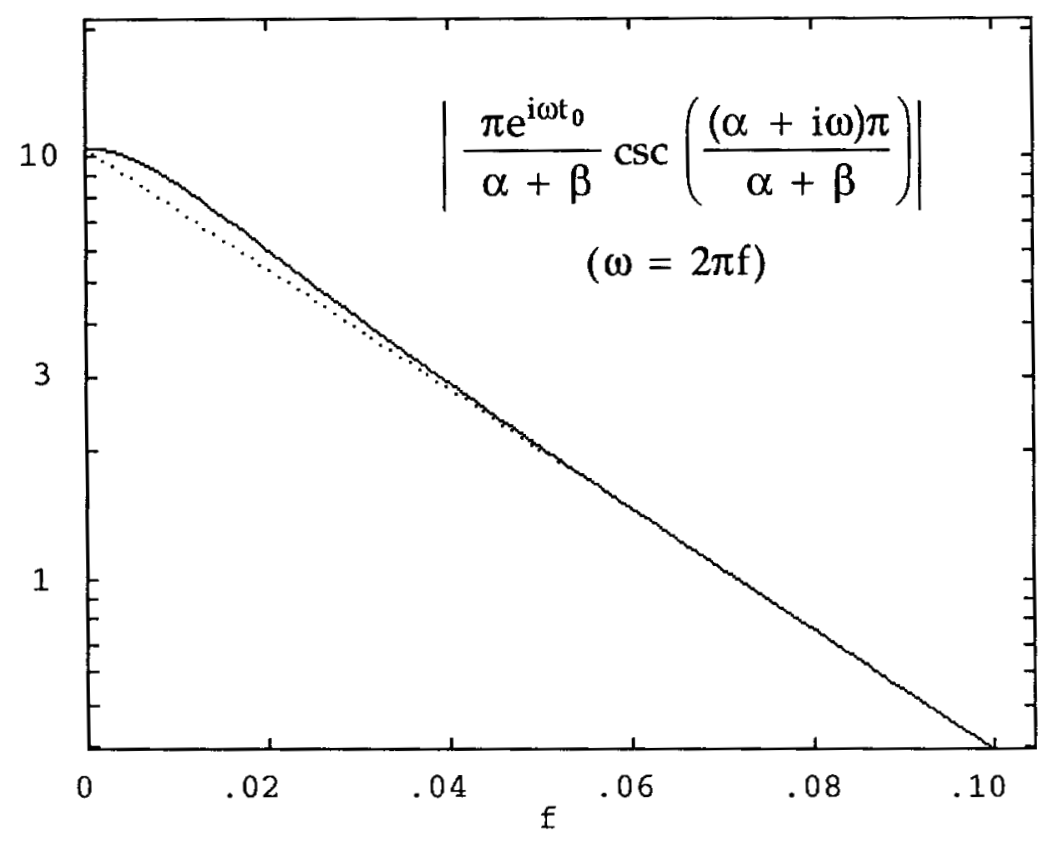

Figure 4. Semi-log plot of the magnitude $|F(\omega)|$ of the Fourier transform of the inverse biexponential function $f(t)$, using $\alpha=0.5$ and $\beta=0.1$. The highfrequency asymptote $2 \xi \mathrm{e}^{-\xi \omega}$, where $\xi=\pi /(\alpha+\beta)$, is shown by the dotted line. 


\section{Sum of Two Inverse Biexponential Functions}

A difficulty with the inverse biexponential form is that if $\alpha \gg \beta$ (or vice versa), then the asymptote associated with $\alpha$ (or $\beta$ ) is not a good representation of the leading (or trailing) edge profile, as suggested by Figure 1. One way of overcoming this problem is to model the pulse as the sum of two inverse biexponential functions, where the leading edge of the first and the trailing edge of the second become the leading and trailing profiles of the pulse, and the trailing edge of the first and the leading edge of the second overlap in such a way as to provide a smooth transition from one function to another. In this way both the leading and trailing edges can be made to follow exponential profiles much more closely than in the case of the single inverse biexponential function. This pulse sum is represented in the time domain as

$$
f(t)=f_{1}(t)+f_{2}(t)=\frac{1}{e^{-\alpha_{1}\left(t-t_{1}\right)}+e^{\beta_{1}\left(t-t_{1}\right)}}+\frac{\gamma}{e^{-\alpha_{2}\left(t-t_{2}\right)}+e^{\beta_{2}\left(t-t_{2}\right)}}
$$

where the $\alpha$ 's and $\beta$ 's are positive real numbers. $\alpha_{1}$ and $-\beta_{2}$ are the exponential slopes of the leading and trailing edges, $-\beta_{1}$ and $\alpha_{2}$ are the exponential slopes of the overlapping edges, $t_{1}$ and $t_{2}$ are where the asymptotes for each pulse intersect, and $\gamma$ determines the amplitude of the second pulse relative to the first.

We illustrate these concepts by plotting an example pair of biexponential functions, $f_{1}(t)$ and $f_{2}(t)$, and their sum $f(t)=f_{1}(t)+f_{2}(t)$, in Figure 5 (on log-linear axes) and in Figure 6 (on linear-linear axes), using the following parameter values (in arbitrary units):

$$
\begin{array}{lr}
\alpha_{1}=0.5 & \alpha_{2}=2.7 \\
\beta_{1}=3 & \beta_{2}=0.1 \\
t_{1}=5 & t_{2}=5.2
\end{array}
$$

The leading and trailing edge asymptotes for the sum are also plotted in both Figures. This choice of parameters resulted in a very smooth sum function where the transition region between the two biexponentials is narrower than the leading and trailing edge regions. That is, the values for $\beta_{1}$ and $\alpha_{2}$ are much larger than those for $\alpha_{1}$ and $\beta_{2}$, and the asymptote intersection time difference $t_{2}-t_{1}$ is small. A comparison of the graphs in Figures 1 and 5 -- which have the same horizontal and vertical axes and (except for a slight time shift) the same leading and trailing asymptote functions -- shows that a sum of two overlapping biexponential functions can indeed follow the leading and trailing edge asymptotes much more closely than a single biexponential function can. Other values of the parameters will of course result in different shape effects. For example, a "flat-top" pulse can be obtained by suitably separating $t_{1}$ and $t_{2}$ and adjusting the other parameters. 


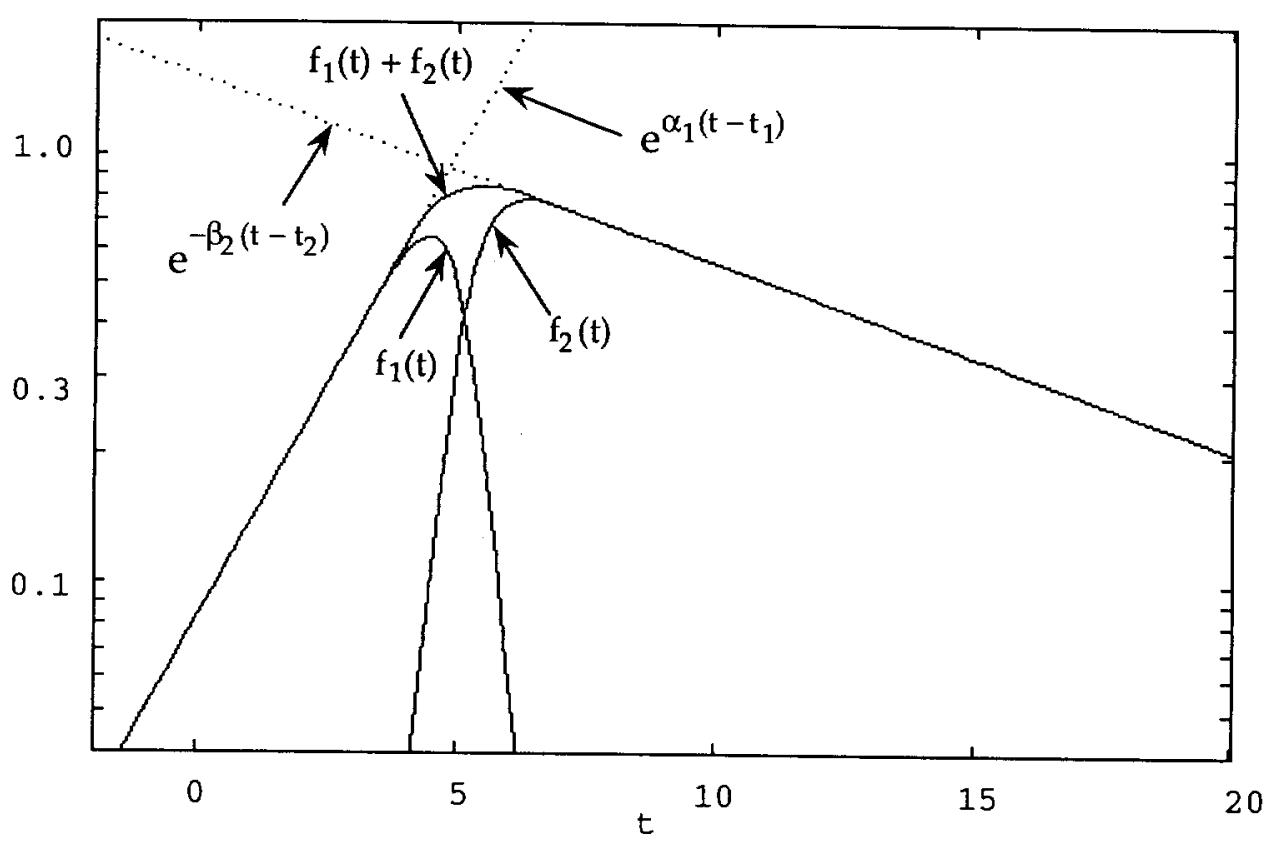

Figure 5. Log-linear plot of two inverse biexponential functions $f_{1}(t)$ and $f_{2}(t)$ and their sum, as discussed in the text. The leading and trailing edge asymptotes are also plotted.

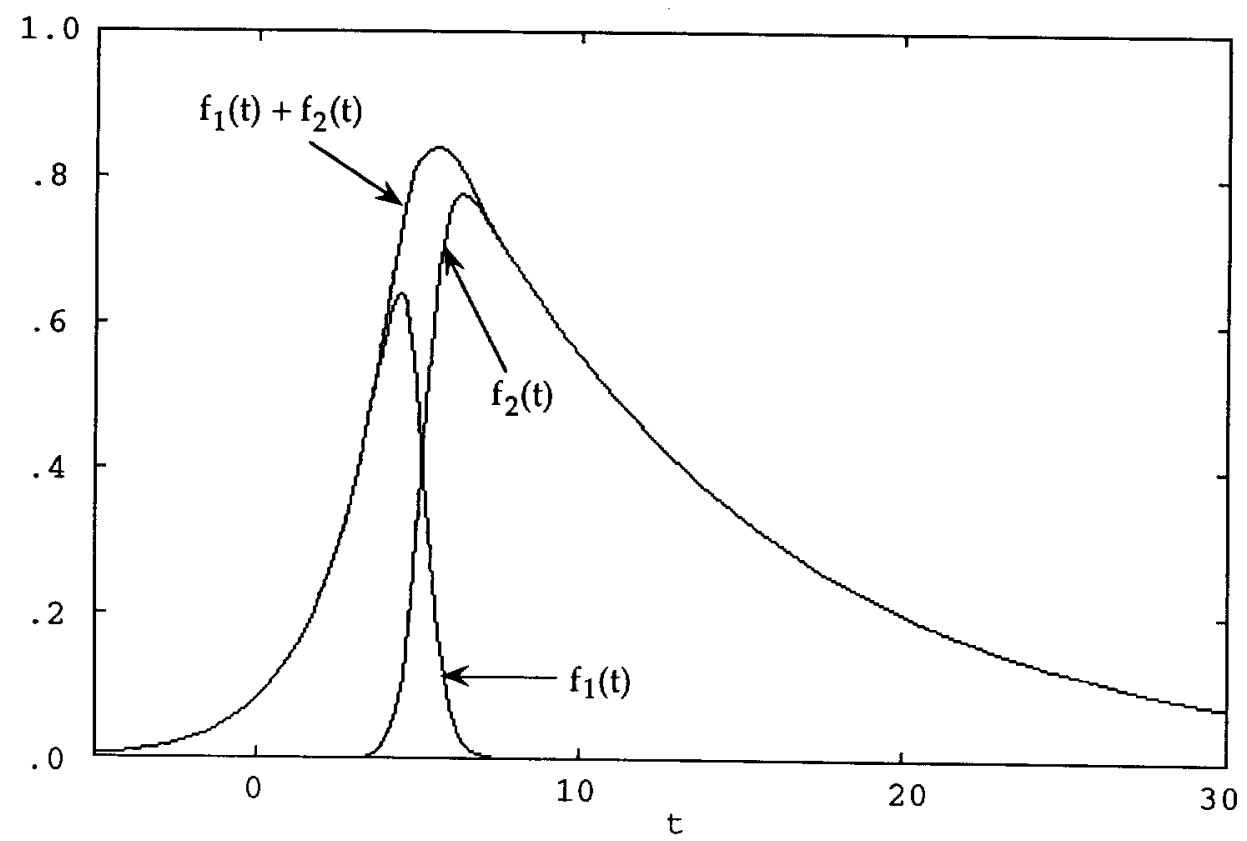

Figure 6. Linear-linear plot of two inverse biexponential functions $f_{1}(t)$ and $f_{2}(t)$, and their sum, as discussed in the text. 
The Fourier transform of the sum of biexponential functions is, of course, the sum of the Fourier transforms of the individual functions, and is given by

$$
F(\omega)=F_{1}(\omega)+F_{2}(\omega),
$$

where the component transforms $F_{1}(\omega)$ and $F_{2}(\omega)$ are the Fourier transforms of $f_{1}(t)$ and $f_{2}(t)$, which in turn are given by

$$
F_{i}(\omega)=\xi_{i} e^{i \omega t_{i}} \csc \left(\left(\alpha_{i}+i \omega\right) \xi_{i}\right),
$$

where $\xi_{i}=\pi /\left(\alpha_{i}+\beta_{i}\right)$, for $i=1$ and 2 . Note that the phase relation between each

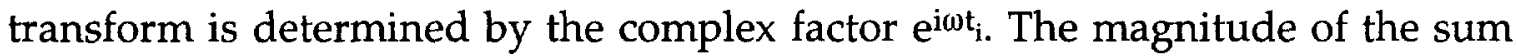
transform $|F(\omega)|$ is the magnitude of the sum of the individual transforms, that is, $|F(\omega)|=\left|F_{1}(\omega)+F_{2}(\omega)\right|$. The magnitudes $\left|F_{1}(\omega)\right|,\left|F_{2}(\omega)\right|$ and $|F(\omega)|$ are shown plotted in Figures 7,8 , and 9, using the parameter values given previously for $f_{1}(t)$ and $f_{2}(t)$. The magnitudes $\left|F_{1}(\omega)\right|$ and $\left|F_{2}(\omega)\right|$ are shown by dotted lines. The axes in Figure 7 are logarithmic, while log-linear axes are used in Figures 8 and 9. Figure 9 is an expanded view of the upper left corner region of Figure 8, which more clearly shows how the different magnitudes vary with frequency. (In all plots $\omega=2 \pi \mathrm{f}$.)

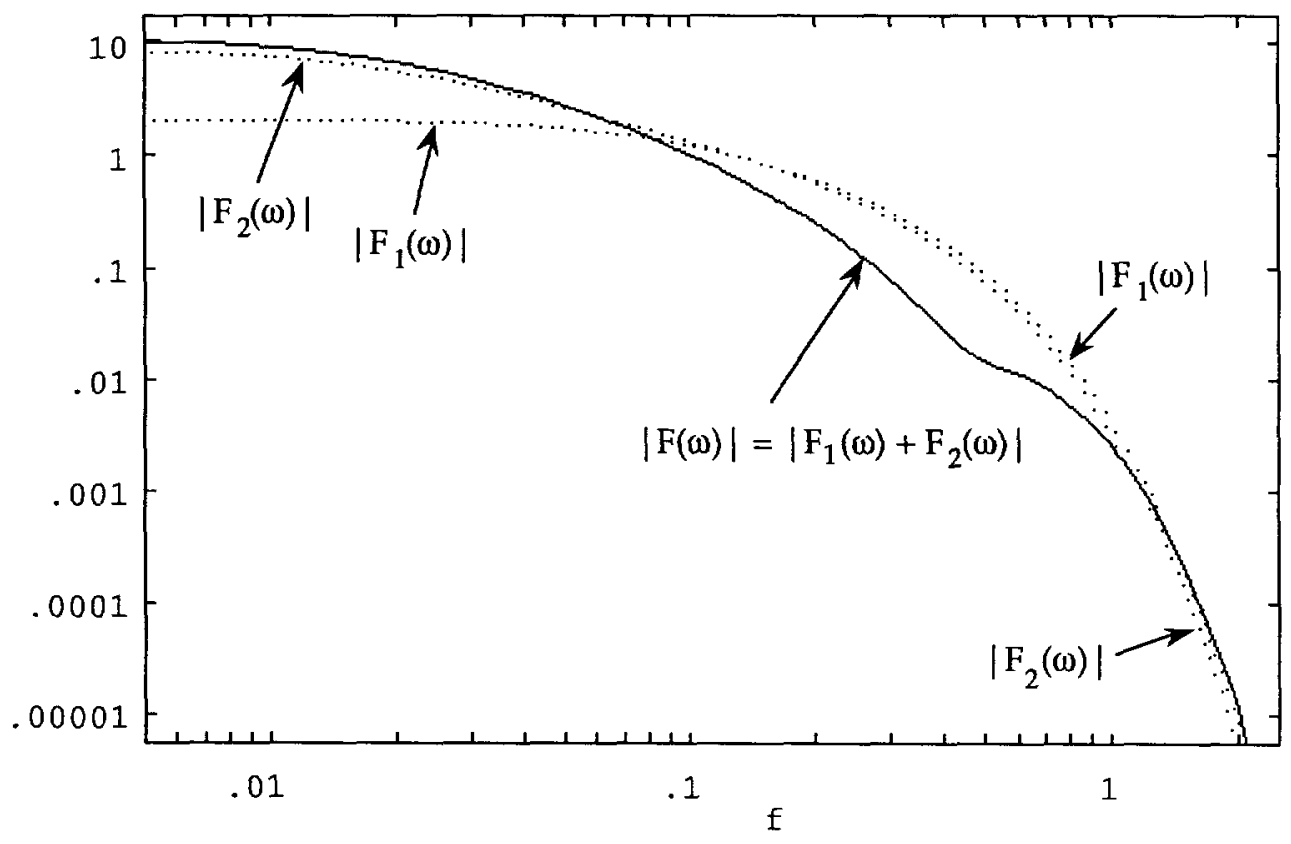

Figure 7. Log-log plot of the magnitudes of the Fourier transforms of the individual and sum inverse biexponential functions shown in Figures 5 and 6. $F_{1}(\omega)$ and $F_{2}(\omega)$ are Fourier transforms of $f_{1}(t)$ and $f_{2}(t)$ respectively. 


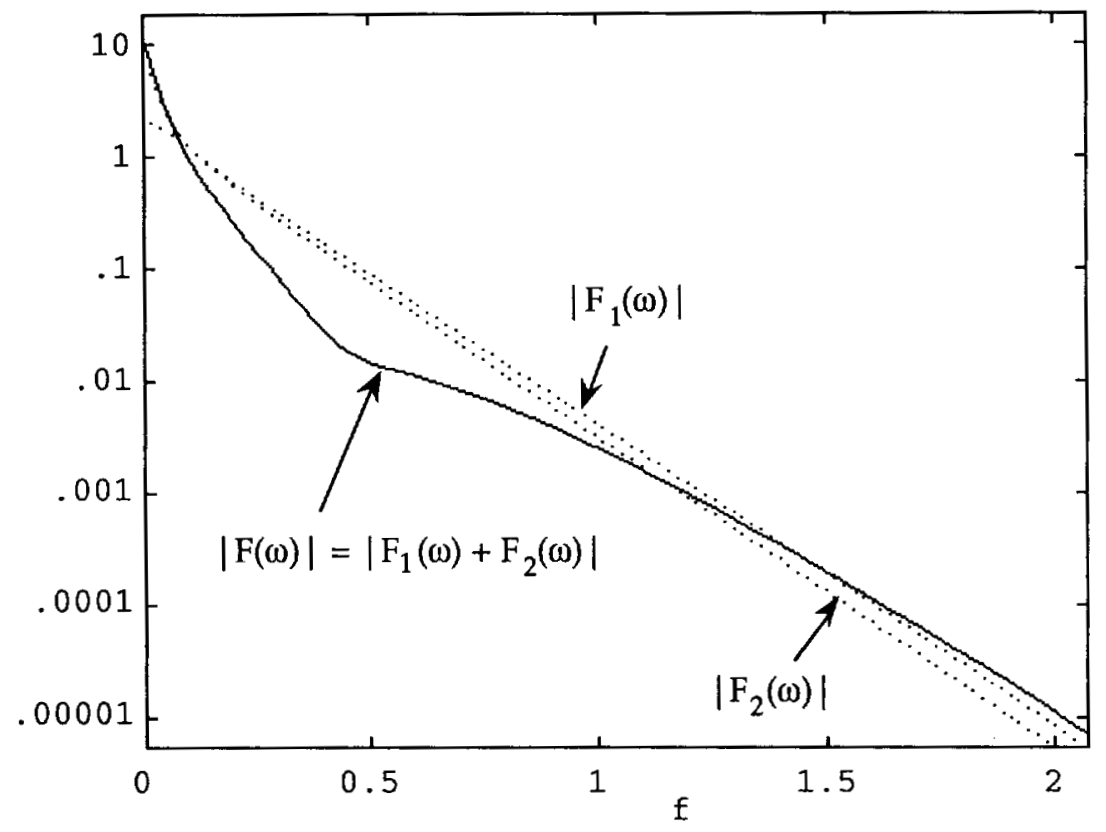

Figure 8. Semi-log plot of the Fourier transform magnitudes for the individual and sum inverse biexponential functions of Figures 5 and 6.

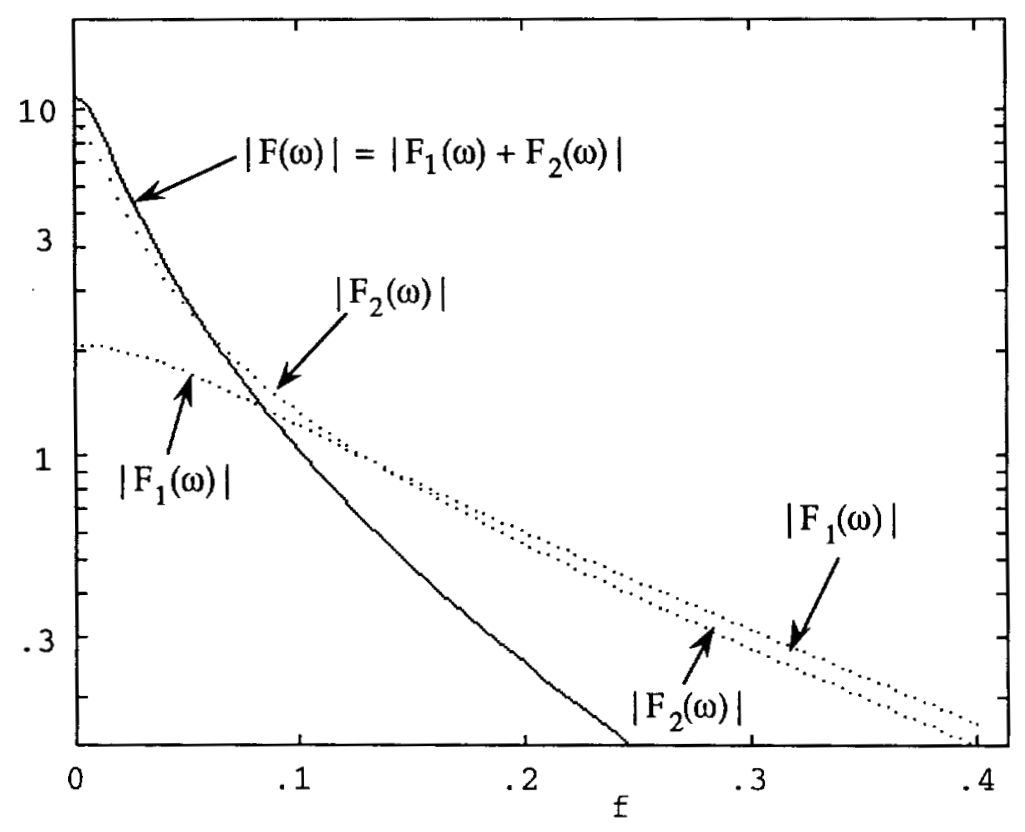

Figure 9. Expanded view of upper left region of Figure 8.

The remarkable feature of the magnitude of the sum transform $F(\omega)$ for the parameters used here is that it dips considerably below the individual magnitudes of 
the transforms $F_{1}(\omega)$ and $F_{2}(\omega)$ over part of the frequency range. This produces the tilted shallow trough around $\mathrm{f}=0.4$ shown in Figures 7 and 8 . This dip is the result of the frequency domain phasing interference between the two biexponential components that occurs because their time-domain peaks occur at different times. (Recall that the relative phase of each component is manifested as the complex

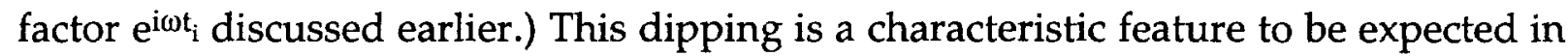
Fourier transforms of multicomponent functions when the components occur at different times.

To illustrate this phenomenon more fully, we plot on log-linear axes in Figure 10 the magnitudes of the Fourier transform of the sum of the biexponentials for different asymptote crossover time separations $\left(t_{2}-t_{1}\right)=0.4,0.8$ and 1.2 of the two biexponentials. This was done by setting $t_{2}=5.4,5.8$ and 6.2 in the sum while keeping all the other parameters unchanged. The magnitude for $\left(t_{2}-t_{1}\right)=0.2$ was plotted previously in Figure 8, and can be included in the comparison. It is easy to see that as the time separation $\Delta t=t_{2}-t_{1}$ between the two component functions increases, the frequency interval $\Delta \mathrm{f}$ between the dips in the transforms decreases. One finds (and can verify analytically) that this frequency spacing and the time separation interval follows the simple "uncertainty principle" law $\Delta f \Delta t=1$ for each transform case.

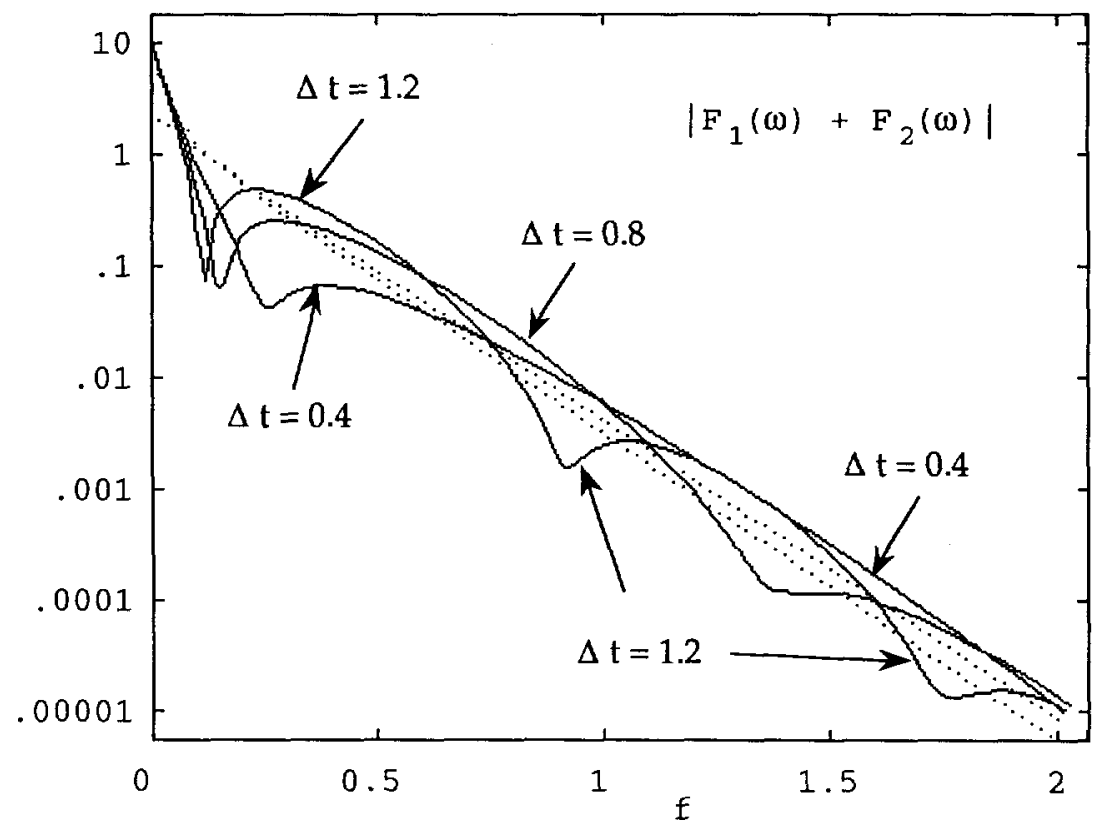

Figure 10. Magnitude $\left|F_{1}(\omega)+F_{2}(\omega)\right|$ of the sum of the Fourier transforms of the inverse biexponential functions $f_{1}(t)$ and $f_{2}(t)$ for different time separations $t_{2}-t_{1}$ of these components. See text for details. The dotted curves are $\left|F_{1}(\omega)\right|$ and $\left|F_{2}(\omega)\right|$ as in Figure 8. 
5. A Fractal Family of Inverse Biexponential Functions

A suite of pulse functions that have the same exponential leading and trailing edge asymptotes $e^{\alpha\left(t-t_{0}\right)}$ and $e^{-\beta\left(t-t_{0}\right)}$ can be formed by applying a semilogarithmic "magnification" transformation to the inverse biexponential function. This consists of dividing the dependent variable $\left(t-t_{0}\right)$ by a positive factor $\kappa$, and raising the resulting inverse biexponential function to the power of this same factor $\kappa$. The resulting function is given by

$$
f_{\kappa}(t)=\frac{1}{\left(e^{-\alpha\left(t-t_{0}\right) / \kappa}+e^{\beta\left(t-t_{0}\right) / \kappa}\right)^{\kappa}} .
$$

This transformation has the same effect as scaling the $\ell n f_{K}$ and $\left(t-t_{0}\right)$ axes of a plot by the same factor $1 / \kappa$, thus producing a "zoom" effect for $\kappa \neq 1$. Thus, if one displays $\left(f_{\kappa}(t)\right)^{1 / \kappa}$ versus $\left(t-t_{0}\right) / \kappa$ in a log-linear plot, the plotted shape of the function is invariant for all $\kappa>0$ (and is the same as that for $\kappa=1$, an example of which is shown in Figure 1). This invariance gives $f_{k}(t)$ a fractal character.

In Figure 11 we plot $f_{k}(t)$ for $k=1 / 8,1 / 4,1 / 2,1,2$ and 4 (using $\alpha=0.5, \beta=0.1$ and $t_{0}=5$ ) with semi-log axes. This display shows that these curves all have the same pair of asymptotes, and as $\kappa$ diminishes the function $f_{K}(t)$ more closely approaches these intersecting asymptotes as a limit. This suite of curves is replotted in Figure 12 using linear-linear axes, where it is seen that $f_{k}(t)$ becomes narrower and more sharply peaked in the neighborhood of $t_{0}$ as $\mathrm{K}$ decreases.

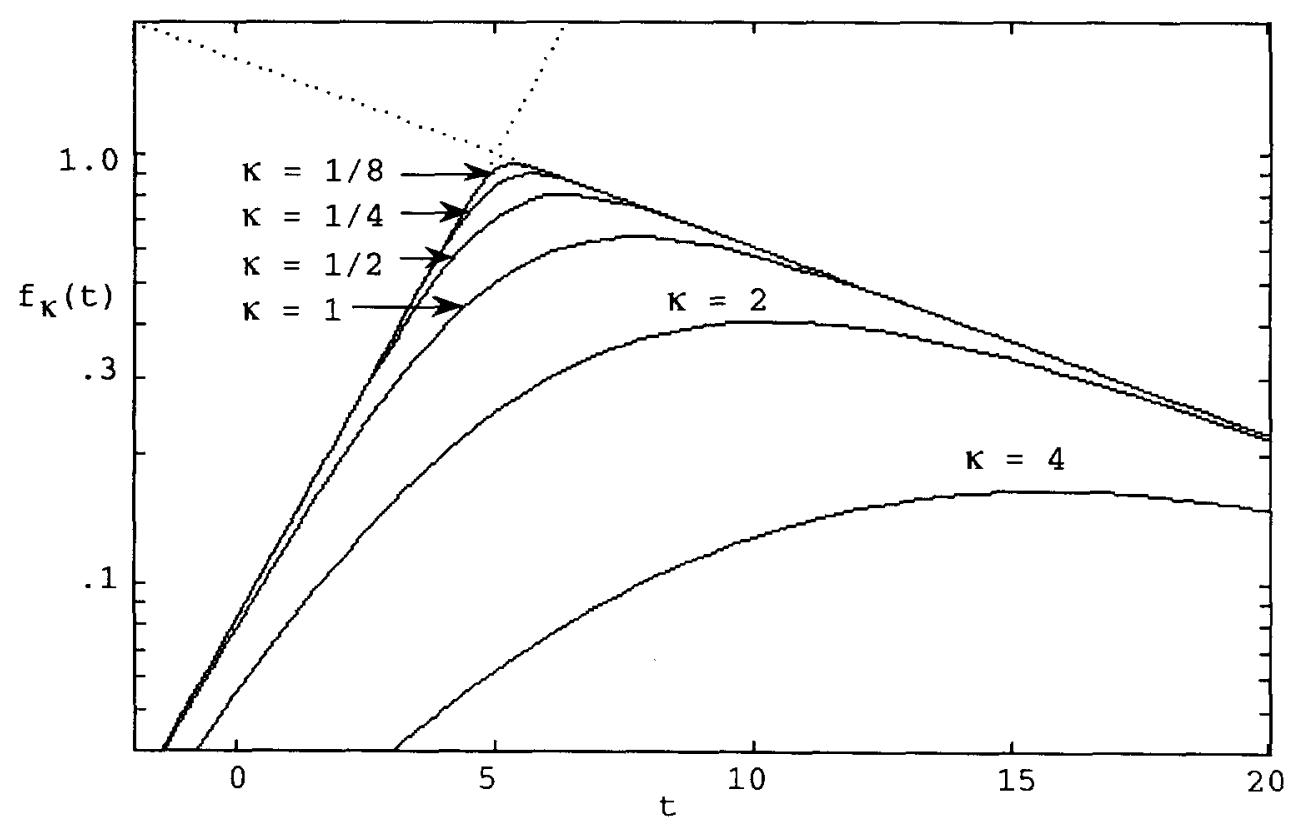

Figure 11. Semi-log plot of the fractal inverse biexponentials $\mathrm{f}_{\mathrm{K}}(\mathrm{t})$ for $\alpha=0.5$, $\beta=0.1, t_{0}=5$. The common asymptotes $e^{\alpha\left(t-t_{0}\right)}$ and $e^{-\beta\left(t-t_{0}\right)}$ are shown dotted. 


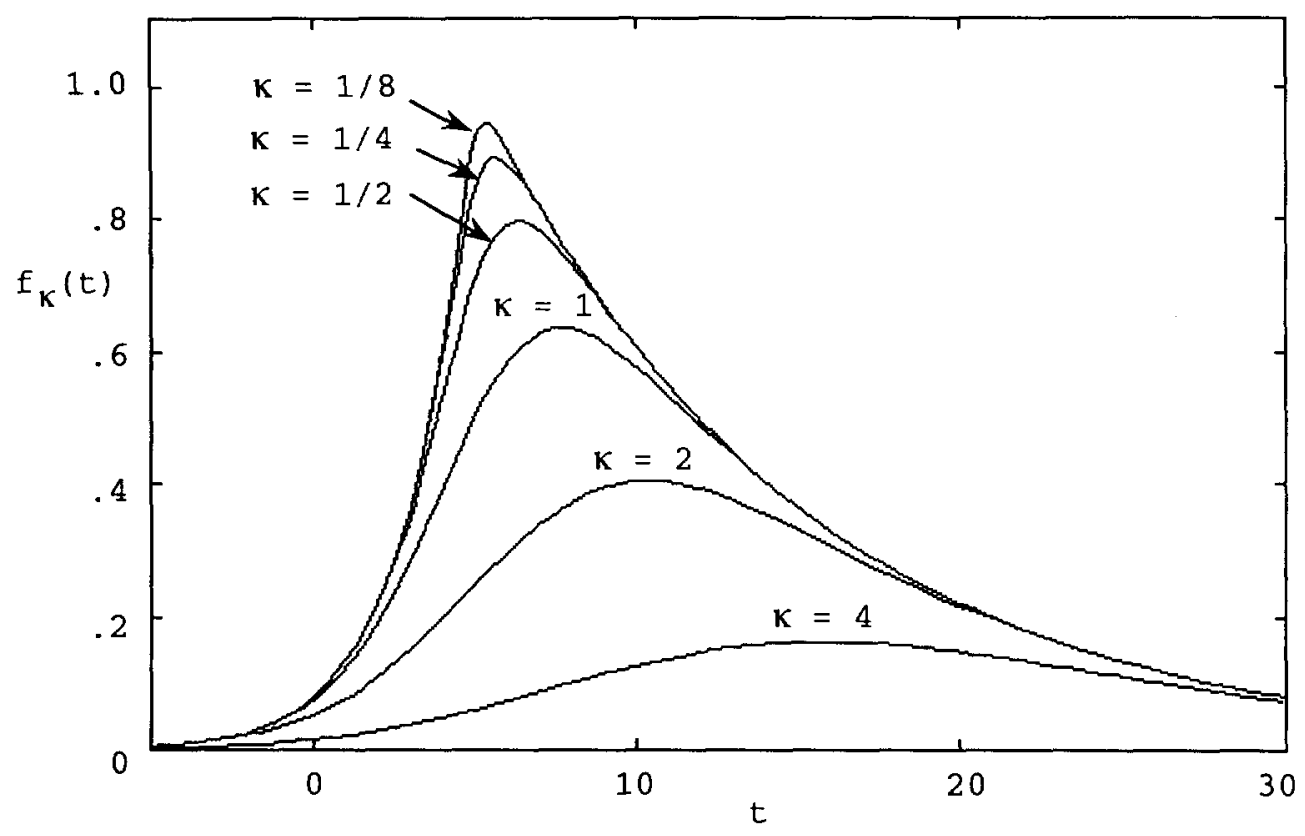

Figure 12. Linear plot of the fractal inverse biexponential functions $\mathrm{f}_{\mathrm{K}}(\mathrm{t})$ for $\alpha=0.5, \beta=0.1, \mathrm{t}_{0}=5$.

The Fourier transform $F_{K}(\omega)$ of $f_{K}(t)$ is obtained making variable substitutions $x=(\alpha+\beta)\left(t-t_{0}\right) / \kappa$ and $\lambda=(\alpha+i \omega) \kappa /(\alpha+\beta)$, as follows:

$$
F_{k}(\omega)=\int_{-\infty}^{+\infty} f_{k}(t) e^{i \omega t} d t=\frac{\kappa e^{i \omega t} t_{0}}{\alpha+\beta} \int_{-\infty}^{+\infty} \frac{e^{\lambda x} d x}{\left(1+e^{x}\right)^{\mathrm{x}}}
$$

These algebraic transformations are similar to those carried out in Section 3 for the original $(\kappa=1)$ inverse biexponential case. The above integral has been evaluated in Section 2; on setting $\mu=1$ there we obtain the Fourier transform as

$$
F_{K}(\omega)=\frac{\kappa e^{i \omega t_{0}}}{\alpha+\beta} \frac{(1-\lambda)(2-\lambda) \ldots . .(\kappa-1-\lambda)}{(\kappa-1) !} \frac{\pi}{\sin \pi \lambda} .
$$

The Fourier transforms for the cases $\kappa=1,2$ and 3 are

and

$$
\begin{gathered}
F_{1}(\omega)=\frac{\pi e^{i \omega t_{0}}}{\alpha+\beta} \csc \left(\frac{\pi(\alpha+i \omega)}{\alpha+\beta}\right) \\
F_{2}(\omega)=\frac{2 \pi e^{i \omega t_{0}}}{(\alpha+\beta)^{2}}(\beta-\alpha-2 i \omega) \csc \left(\frac{2 \pi(\alpha+i \omega)}{\alpha+\beta}\right),
\end{gathered}
$$




$$
F_{3}(\omega)=\frac{3 \pi e^{i \omega t_{0}}}{2(\alpha+\beta)^{3}}(\beta-2 \alpha-3 i \omega)(2 \beta-\alpha-3 i \omega) \csc \left(\frac{3 \pi(\alpha+i \omega)}{\alpha+\beta}\right)
$$

The magnitudes $\left|F_{K}(\omega)\right|$ of these transforms are plotted in Figure 13 using the same parameter values $\alpha=0.5$ and $\beta=0.1$ as for Figures 1 through 4,11 and 12 .

We also include in Figure 13 a plot of the magnitude of the Fourier transform $\mathrm{F}_{0}(\omega)$ of the piecewise continuous function $\mathrm{f}_{0}(\mathrm{t})$ composed of the leading and trailing edge asymptote functions $e^{\alpha\left(t-t_{0}\right)}$ for $t \leq t_{0}$, and $e^{-\beta\left(t-t_{0}\right)}$ for $t \geq t_{0}$. This transform is

$$
F_{0}(\omega)=e^{i \omega t_{0}}\left(\frac{1}{\alpha+i \omega}+\frac{1}{\beta-i \omega}\right)
$$

The curve representing $\left|F_{0}(\omega)\right|$ is labeled " $\kappa=0$ " in the Figure. This follows the notion suggested by Figures 11 and 12 that the piecewise function $\mathrm{f}_{0}(t)$ just defined is in a sense the limiting curve that results from letting the parameter $\mathrm{K}$ of the fractal biexponential function $\mathrm{f}_{\mathrm{K}}(\mathrm{t})$ approach zero.

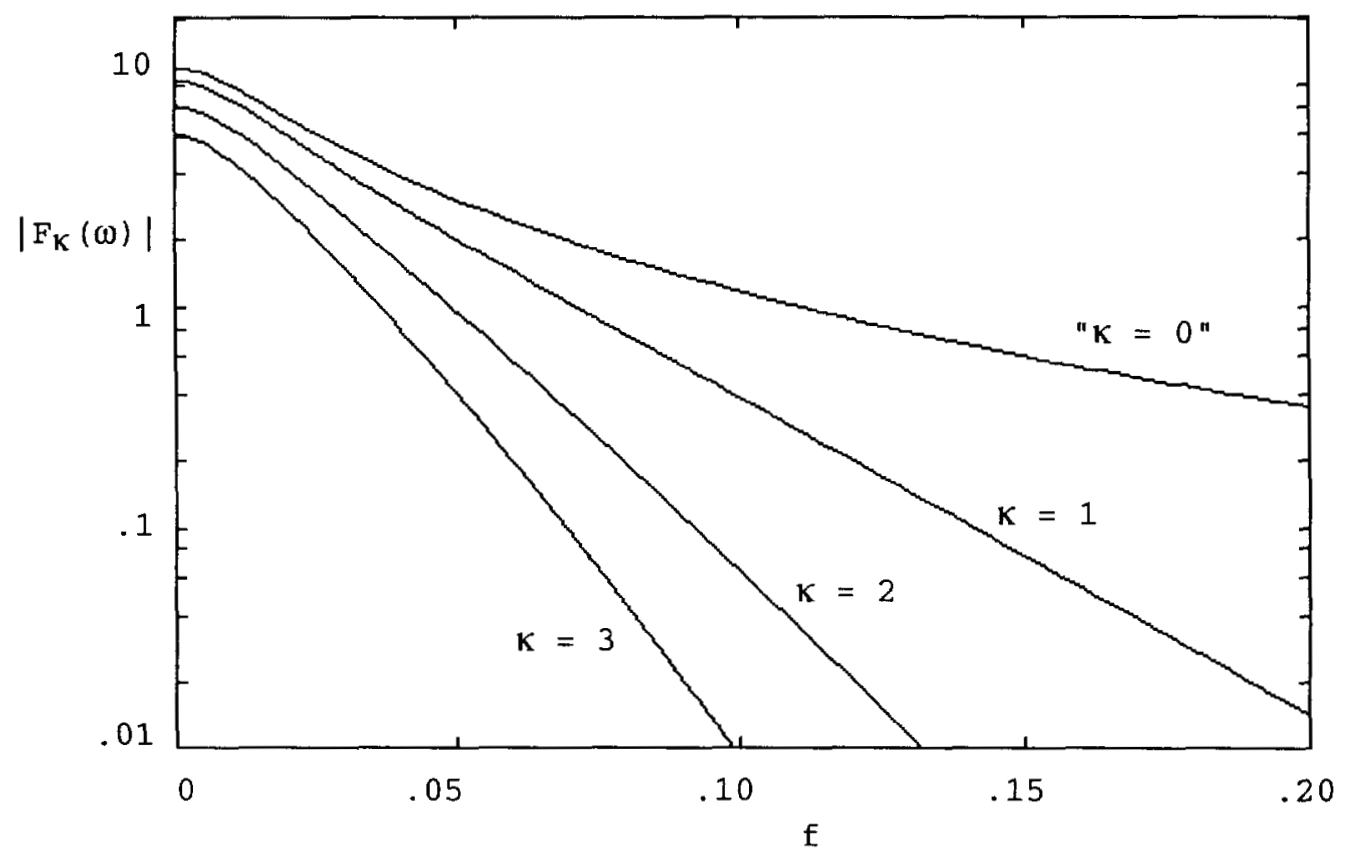

Figure 13. Log-linear plots of the magnitude $\left|F_{K}(\omega)\right|$ of the Fourier transform of the fractal inverse biexponential function $f_{K}(t)$ for integer values of $\kappa$, using parameters $\alpha=0.5$ and $\beta=0.1$.

It turns out that the formalism for the Fourier transform $F_{K}(\omega)$ of $f_{K}(t)$ can be extended to include the case where $\kappa$ is not an integer. This is desirable for the range $0<\kappa<1$ because very sharp functional shapes of $f_{\kappa}(t)$ are obtained that conform 
closely to the leading and trailing asymptotic exponentials. As we showed in Section 2, $\mathrm{k}$ (or $\mathrm{k}$ ) represents the order of the derivative of the integrand of the Fourier transform, and so the use of non-integer $\mathrm{K}$ can involve the notions of fractional derivatives, which have become a well-developed analytical tool (see, e.g., Osler (1971)). This suggests that the extension of $F_{K}(\omega)$ to non-integral $\kappa$ be carried out by employing the gamma function, which has often been used to define the analytic transition between derivative-related polynomials of adjacent orders.

The key recursive property of the gamma function that permits this is given by $\Gamma(z)=(z-1) \Gamma(z-1)=(z-1)(z-2) \Gamma(z-2)=$ etc. Then the polynomial given by $(z-1)(z-2) \ldots . .(z-k+1)$ which has $k-1$ factors can be replaced by the gamma function ratio $\Gamma(\mathrm{z}) / \Gamma(\mathrm{z}-\mathrm{k}+1)$. From this and $\Gamma(\mathrm{n})=(\mathrm{n}-1)$ ! we can formally rewrite the Fourier transform of the fractal biexponential function as

$$
F_{\kappa}(\omega)=\frac{\kappa e^{i \omega t_{0}}}{\alpha+\beta} \frac{\Gamma(\kappa-\lambda)}{\Gamma(1-\lambda) \Gamma(\kappa)} \frac{\pi}{\sin \pi \lambda},
$$

which is a true statement when $\mathrm{K}$ is a positive integer. If we now make use of the relation $\Gamma(\lambda) \Gamma(1-\lambda)=\pi / \sin \pi \lambda$, and the beta function which has the property that $\mathrm{B}(\mathrm{x}, \mathrm{y})=\Gamma(\mathrm{x}) \Gamma(\mathrm{y}) / \Gamma(\mathrm{x}+\mathrm{y})=\mathrm{B}(\mathrm{y}, \mathrm{x})$, we obtain the compact results

$$
F_{\kappa}(\omega)=\frac{\kappa e^{i \omega t_{0}}}{\alpha+\beta} \frac{\Gamma(\kappa-\lambda) \Gamma(\lambda)}{\Gamma(\kappa)}=\frac{\kappa e^{i \omega t_{0}}}{\alpha+\beta} B(\kappa-\lambda, \lambda)
$$

where we recall $\lambda=(\alpha+i \omega) \kappa /(\alpha+\beta)$, and therefore $\kappa-\lambda=(\beta-i \omega) \kappa /(\alpha+\beta)$. The evaluation of $F_{K}(\omega)$ requires calculating $\Gamma(z)$ for complex argument.

Note added in proof. We can indeed verify that this new expression correctly represents the Fourier transform of $f_{K}(t)$ for non-integral $k$, because a definition of the beta function is the integral (Abramowitz and Stegun (1965))

$$
\mathrm{B}(\mathrm{z}, \mathrm{w})=\int_{0}^{+\infty} \frac{\mathrm{t}^{\mathrm{z}-1} \mathrm{dt}}{(1+\mathrm{t})^{\mathrm{z}+\mathrm{w}}}=\frac{\Gamma(\mathrm{z}) \Gamma(\mathrm{w})}{\Gamma(\mathrm{z}+\mathrm{w})} .
$$

On making the substitutions $t=e^{x}$ and $z+w=k$ we find that this relation can be rewritten

$$
\mathrm{B}(\mathrm{z}, \mathrm{\kappa}-\mathrm{z})=\int_{-\infty}^{\infty} \frac{\mathrm{e}^{\mathrm{xz}} \mathrm{dx}}{\left(1+\mathrm{e}^{\mathrm{x}}\right)^{\mathrm{k}}}=\frac{\Gamma(\mathrm{z}) \Gamma(\kappa-\mathrm{z})}{\Gamma(\kappa)},
$$

which is exactly the expression of the generalization of $F_{K}(\omega)$ to non-integer $\kappa$ that was formally derived above. 
We plot in Figure 14 the magnitude $\left|F_{K}(\omega)\right|$ of the Fourier transforms given by this new compact form, for integral and non-integral values of $k$. These plots were produced by calculating each of the three gamma functions indicated, by using a Fortran 77 double precision complex gamma function algorithm that was publicly available on the Internet, and whose accuracy was verified by comparison with the table entries of the gamma function for complex argument presented the handbook edited by Abramowitz and Stegun. The numerical values of $\left|F_{K}(\omega)\right|$ used to plot those curves for which $K$ is an integer are identical to those obtained using the earlier "integer $\mathrm{k}$ " formulas that generated the corresponding curves in Figure 13. We also include in Figure 14 a plot of the magnitude of the Fourier transform $F_{0}(\omega)$ of the piecewise function $f_{0}(t)$ formed from the leading and trailing asymptotic profiles as described earlier; this curve has the label "0".

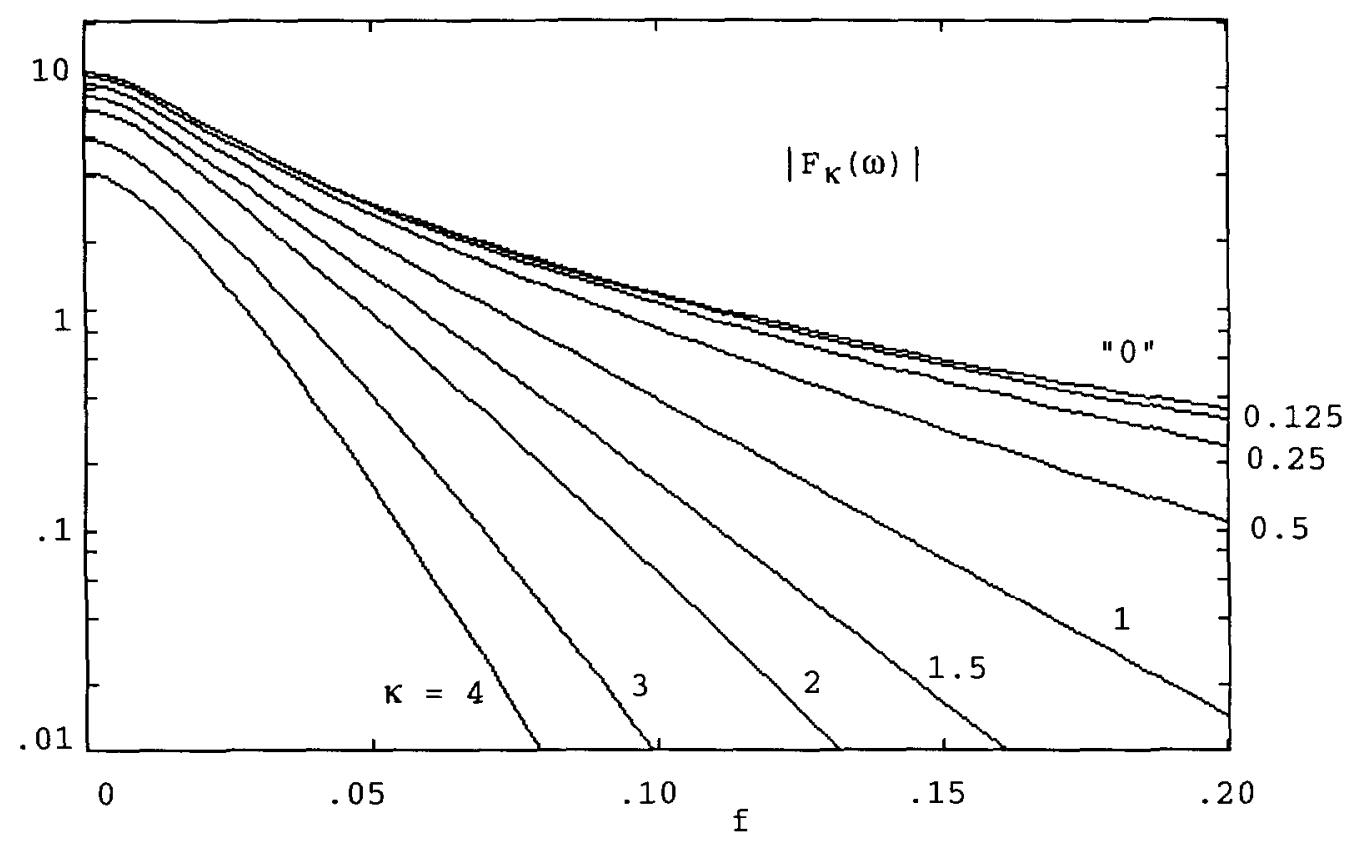

Figure 14. Log-linear plots of the magnitude $\left|F_{K}(\omega)\right|$ of the Fourier transform of $f_{\kappa}(t)$, for integer and non-integer values of $\kappa$, using the parameters $\alpha=0.5$ and $\beta=0.1$. $\left|F_{K}(\omega)\right|$ was calculated from the new Fourier transform formula for $F_{K}(\omega)$ derived on the previous page.

It is clear from these plots that the new generalized form of $F_{K}(\omega)$ is a very satisfactory representation of the Fourier transform of the fractal biexponential function $f_{k}(t)$ for both integral and nonintegral $\kappa$. We see from Figures 11 through 14 that as $K$ approaches zero, the behavior of both this function and its transform smoothly approach that of their respective envelopes, which are the piecewise continuous function formed from the two asymptotic profiles and its Fourier transform; the latter is labeled as $\mathrm{k}=0$ on the transform plots. 
We now derive the asymptotic high-frequency functional form of $\left|F_{K}(\omega)\right|$ by considering its limit as $\omega \rightarrow \infty$. We start with $\mathrm{F}_{\mathrm{K}}(\omega)$ as originally derived here for integer $\kappa$ (rather than work with $\Gamma(\kappa-\lambda) \Gamma(\lambda)$ ), because its limiting asymptotic form is also valid for noninteger $\kappa$, and this approach is much easier. From the definition of $\lambda$ it is clear that $\lambda \rightarrow \mathrm{i} \omega \mathrm{K} /(\alpha+\beta)$ as $\omega \rightarrow \infty$, and so the cosecant term approaches the limit function $-2 \mathrm{ie}^{-\pi \omega \kappa} /(\alpha+\beta)$. The polynomial $(1-\lambda)(2-\lambda) \ldots . .(\kappa-1-\lambda)$ in the numerator has $\kappa-1$ factors; its leading term is thus $(-\lambda)^{\kappa-1}=(-i \omega \kappa /(\alpha+\beta))^{\kappa-1}$ and dominates as $\omega \rightarrow \infty$. We also replace $(\kappa-1)$ ! with $\Gamma(\kappa)$. On putting all this together and then taking absolute values, we find that the high frequency limit form of the Fourier transform magnitude is given by

$$
\left|F_{K}(\omega)\right| \rightarrow \frac{2 \pi}{\omega \Gamma(\kappa)}\left(\frac{\omega \kappa}{\alpha+\beta}\right)^{\kappa} \mathrm{e}^{-\pi \omega \kappa /(\alpha+\beta)} .
$$

We plot this limit function in Figure 15 for the same values of $\kappa, \alpha$ and $\beta$ as used in the previous three plots, along with the corresponding full (i.e. normal) Fourier transform magnitudes. The limit functions are shown by dotted curves, while the transform magnitudes are plotted as solid curves. It is seen that both sets of curves do indeed merge at higher values of $\omega$. We observe that this limit form almost has a fractal character itself similar to $f_{K}(t)$, because in it $\omega$ is multiplied by $\kappa$, and the main terms can be regarded as raised to a power of $\kappa$.

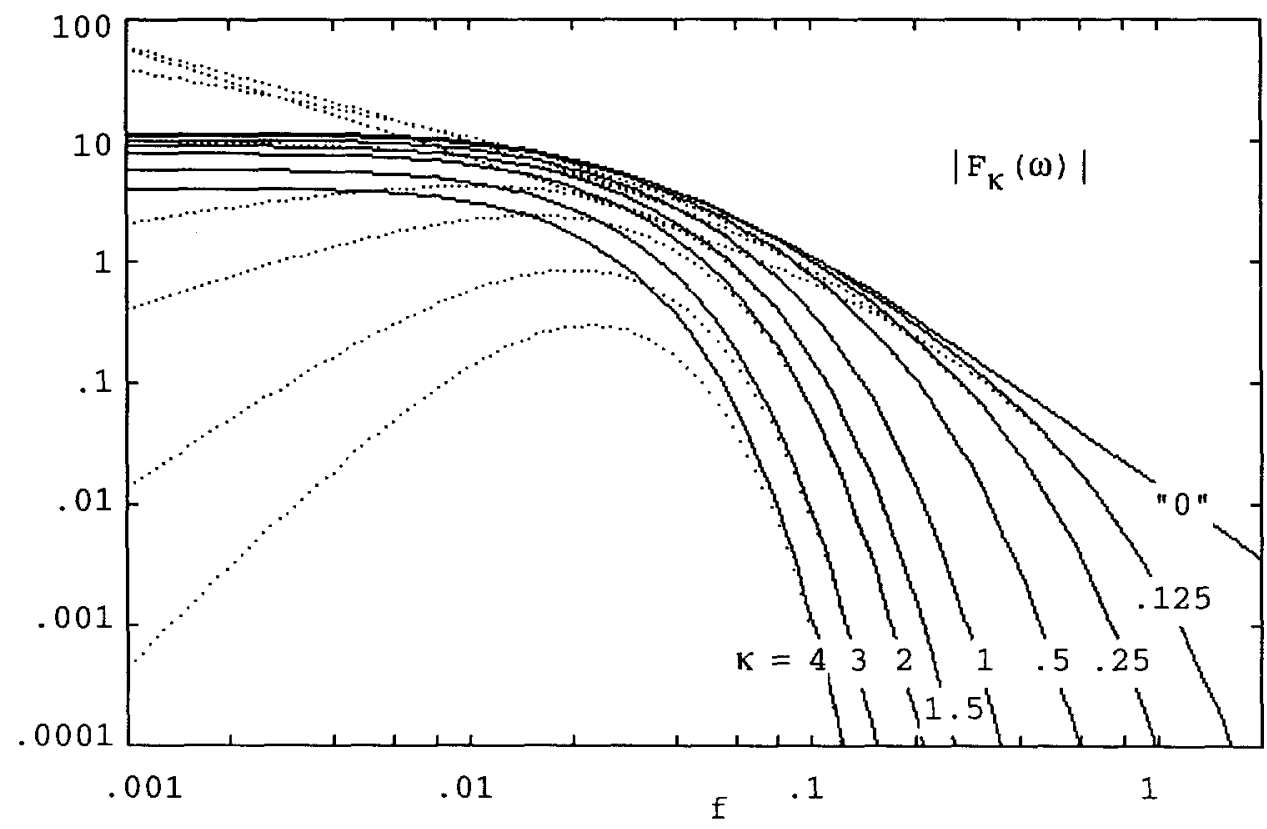

Figure 15. Log-log plots of the magnitude $\left|F_{K}(\omega)\right|$ of the Fourier transform of $f_{K}(t)$, for integer and non-integer values of $\kappa$, with parameter values $\alpha=0.5$ and $\beta=0.1$. The dotted curves are asymptotic high frequency limiting functions corresponding to each value of $\kappa$. 


\section{Bipolar Forms of Inverse Biexponential Functions}

We turn now to bipolar pulses having exponential leading and trailing profiles. The simplest such forms are the first derivative $\mathrm{df}(\mathrm{t}) / \mathrm{dt}$ of the inverse biexponential function $f(t)$, and the function $f(t)$ multiplied by a linear factor $\left(t-t_{1}\right)$. Because the zero crossing of the linear factor at $t_{1}$ can be placed anywhere relative to the inverse biexponential peak near $t_{0}$, the latter function has more versatility.

We treat the derivative function $f^{\prime}(t)=d f(t) / d t$ first. From the definition of $f(t)$ given in Section 3, this is expressed by

$$
f^{\prime}(t)=\frac{\alpha e^{-\alpha\left(t-t_{0}\right)}-\beta e^{\beta\left(t-t_{0}\right)}}{\left(e^{-\alpha\left(t-t_{0}\right)}+e^{\beta\left(t-t_{0}\right)}\right)^{2}} .
$$

Both $f(t)$ and $f^{\prime}(t)$ are shown plotted in Figure 16 (on linear-linear axes) and Figure 17 (on $\log$-linear axes) with parameters $\alpha=1, \beta=0.5$ and $t_{0}=5$. It is seen that $\left|\mathrm{f}^{\prime}(\mathrm{t})\right|$ has the same asymptotic exponential behavior as $f(t)$ for large values of $|t|$. The zero of $f^{\prime}(t)$ occurs at the peak of $f(t)$, which was previously evaluated in Section 3 .

The Fourier transform of the derivative function $f^{\prime}(t)$ is obtained trivially because it is just the Fourier transform $F(\omega)$ of $f(t)$ multiplied by the factor $-i \omega$. This transform was derived previously in Section 3, and so the Fourier transform of the derivative is

$$
-i \omega F(\omega)=-i \omega \xi \mathrm{e}^{\mathrm{i} \omega \mathrm{t}_{0}} \csc ((\alpha+\mathrm{i} \omega) \xi)
$$

where $\xi=\pi /(\alpha+\beta)$. In Figure 18 we plot the magnitudes of the Fourier transforms of the biexponential function and its derivative as $|F(\omega)|$ and $|\omega F(\omega)|$ respectively. The derivative transform has two obvious features: it is zero at $\omega=0$, and although $|\omega \mathrm{F}(\omega)|$ appears to show exponential decline, its log-linear plot slope approaches that of $|F(\omega)|$ for large $\omega$ only logarithmically.

We finally consider the bipolar pulse obtained by multiplying the inverse biexponential function $f(t)$ by the linear factor $\left(t-t_{1}\right)$. This is written

$$
\left(t-t_{1}\right) f(t)=\frac{t-t_{1}}{e^{-\alpha\left(t-t_{0}\right)}+e^{\beta\left(t-t_{0}\right)}} .
$$

Both $f(t)$ and $\left(t-t_{1}\right) f(t)$ are plotted in Figure 19 (on linear-linear axes) and Figure 20 (on log-linear axes) with the parameters $\alpha=1, \beta=0.5, t_{0}=5$ and $t_{1}=6$. Figure 20 shows that $\left|\left(t-t_{1}\right) f(t)\right|$ has almost, but not quite the same asymptotic exponential behavior as $f(t)$ for large values of $|t|$. 


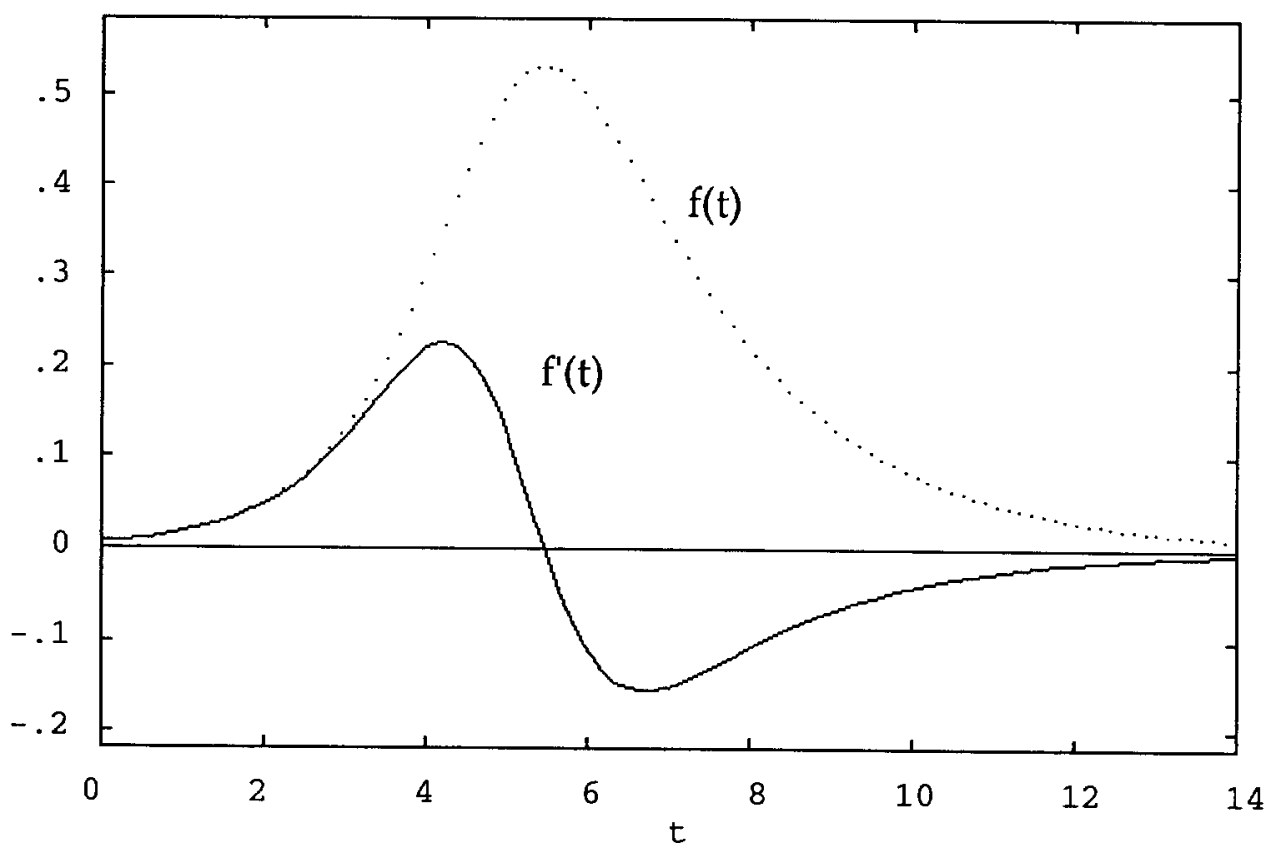

Figure 16. Linear-linear plot of the inverse biexponential function $f(t)$ (shown dotted) and its derivative $f^{\prime}(t)$ (solid) for $\alpha=1, \beta=0.5$ and $t_{0}=5$.

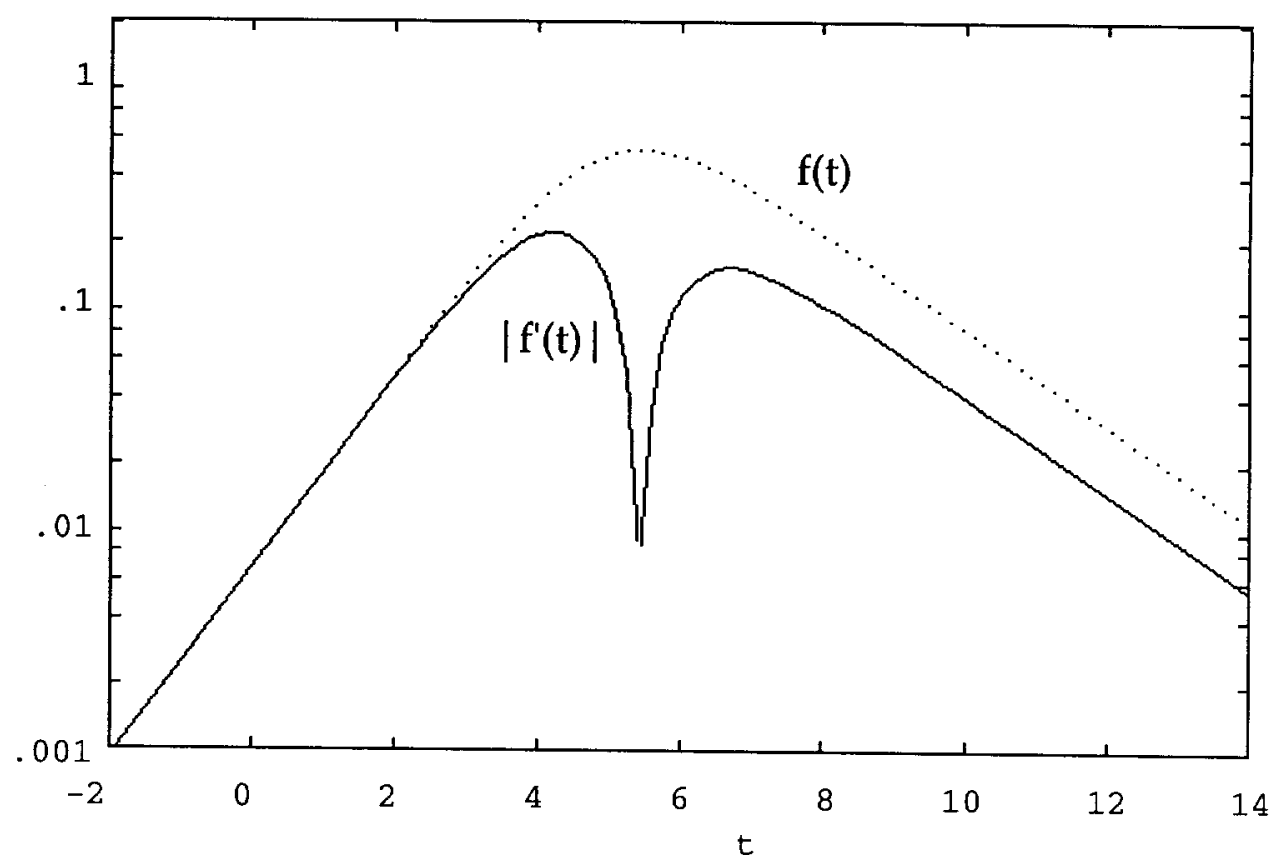

Figure 17. Log-linear plot of the inverse biexponential function $f(t)$ (dotted curve) and the magnitude $\left|f^{\prime}(t)\right|$ of its derivative (solid curve) for $\alpha=1, \beta=0.5$ and $t_{0}=5$. 


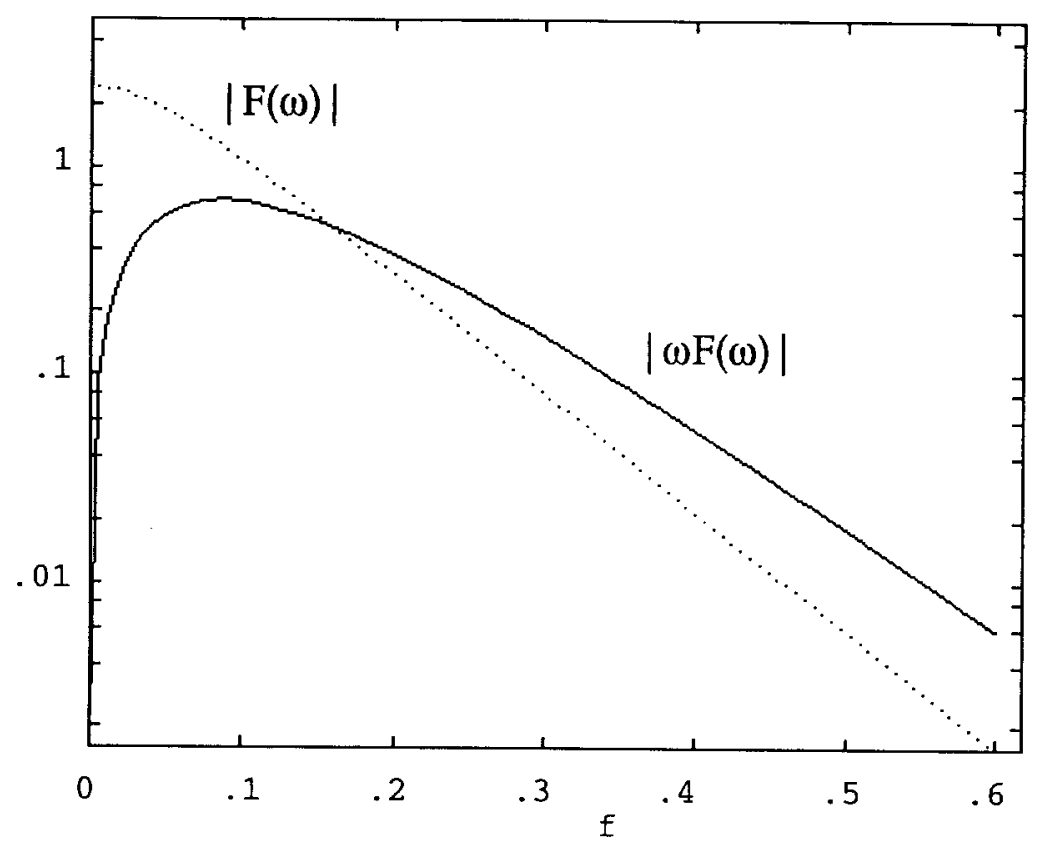

Figure 18. Log-linear plot of the magnitudes of the Fourier transforms of the inverse biexponential function $f(t)$ and its derivative $f^{\prime}(t)$ as $|F(\omega)|$ (shown dotted) and $|\omega F(\omega)|$ (solid).

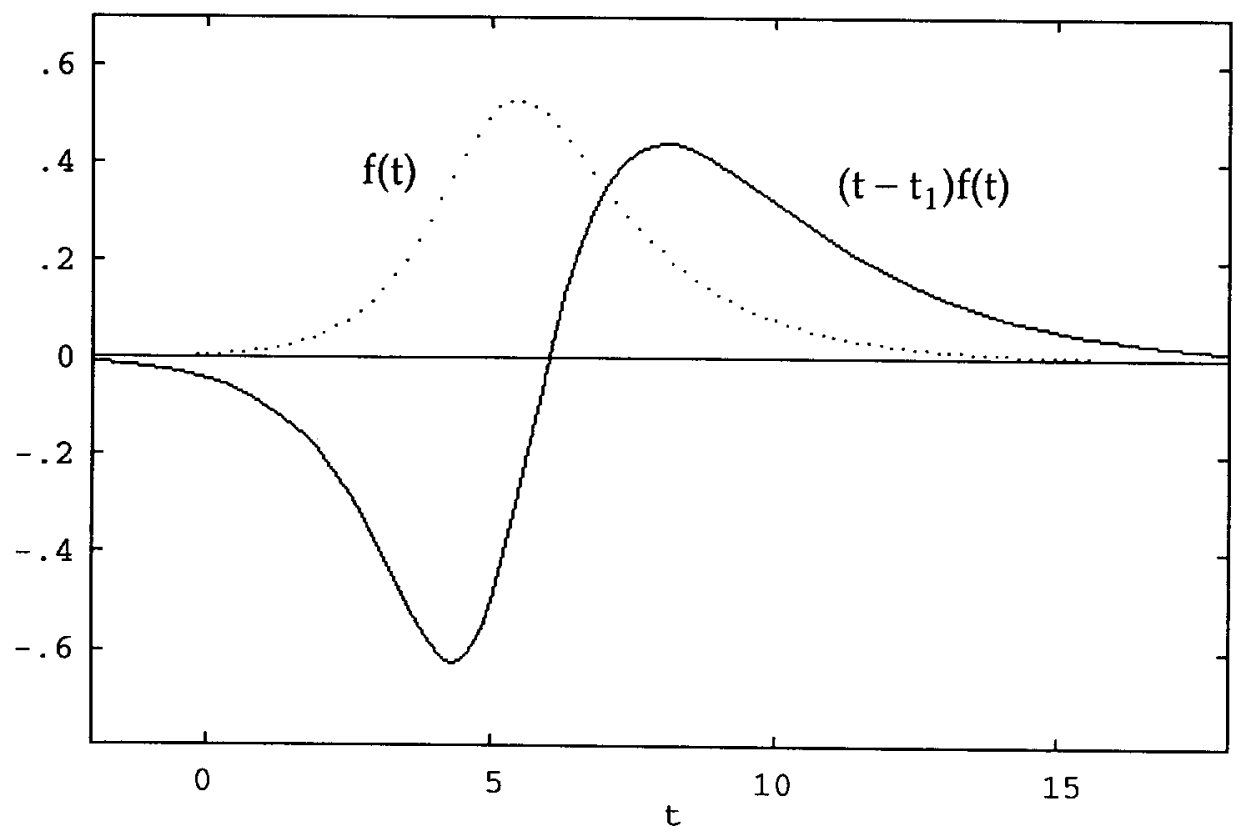

Figure 19. Linear-linear plot of the inverse biexponential function $f(t)$ (dotted curve) and the derived bipolar form $\left(t-t_{1}\right) f(t)$ (solid curve) for $\alpha=1, \beta=0.5 t_{0}=5$ and $t_{1}=6$. 


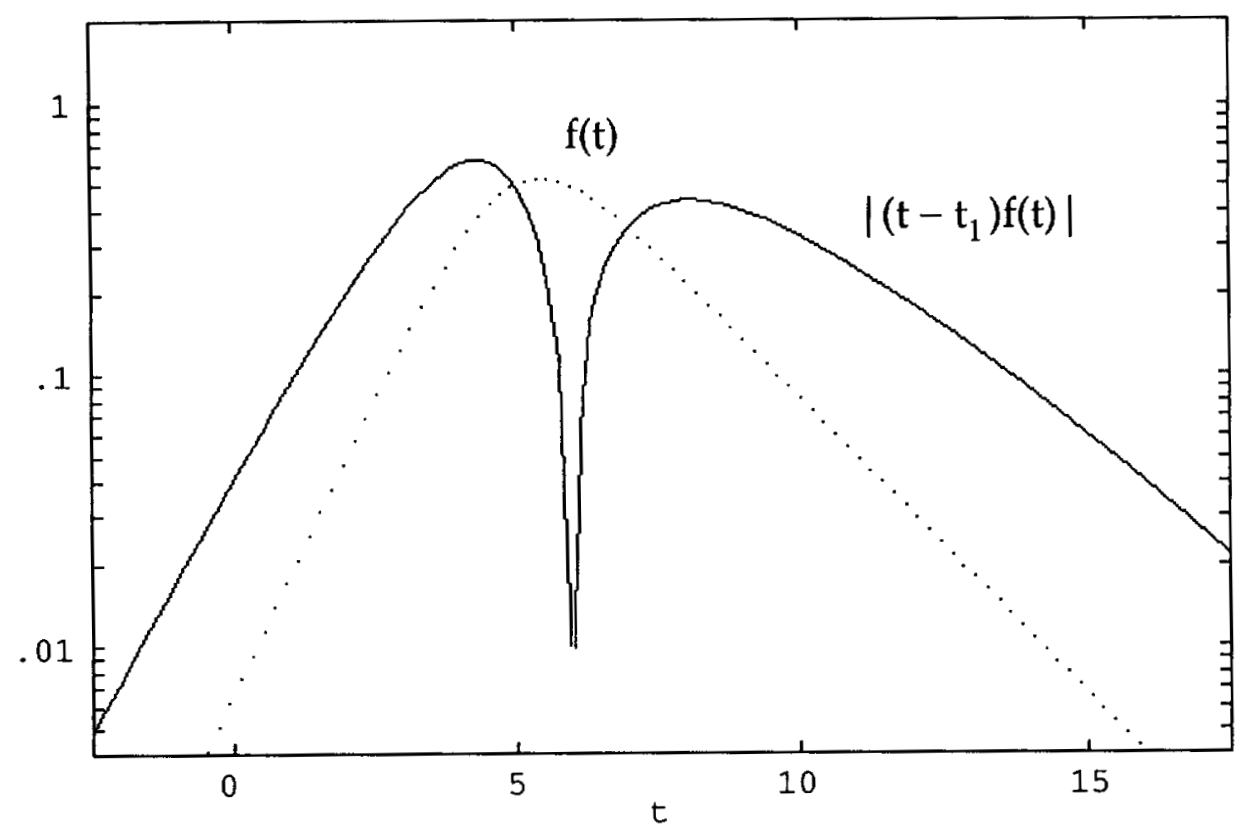

Figure 20. Log-linear plot of the inverse biexponential $\mathrm{f}(\mathrm{t})$ (shown dotted) and magnitude of the bipolar function $\left(t-t_{1}\right) f(t)$ (solid curve) for $\alpha=1, \beta=0.5, t_{0}=5$ and $t_{1}=6$.

The Fourier transform of the bipolar function $\left(t-t_{1}\right) f(t)$ is obtained almost as trivially, because the inverse transform of the derivative $F^{\prime}(\omega)=d F(\omega) / d \omega$ is $+i t f(t)$, by the same reasoning that $-i \omega F(\omega)$ is the Fourier transform of the derivative function $f^{\prime}(t)=d f(t) / d t$. Since $t_{1}$ is a constant, the Fourier transform of $\left(t-t_{1}\right) f(t)$ is therefore

where

$$
F_{\text {bipolar }}(\omega)=-\mathrm{iF}^{\prime}(\omega)-\mathrm{t}_{1} \mathrm{~F}(\omega),
$$

$$
\mathrm{F}(\omega)=\xi \mathrm{e}^{\mathrm{i} \omega \mathrm{t}_{0}} \csc ((\alpha+\mathrm{i} \omega) \xi)
$$

and $\xi=\pi /(\alpha+\beta)$. Carrying out the derivative operation $(d / d \omega)$ gives

$$
F_{\text {bipolar }}(\omega)=\xi \mathrm{e}^{\mathrm{i} \omega \mathrm{t}_{0}} \csc ((\alpha+\mathrm{i} \omega) \xi)\left[\left(\mathrm{t}_{0}-\mathrm{t}_{1}\right)-\xi \cot ((\alpha+\mathrm{i} \omega) \xi)\right] .
$$

This result could also have been obtained by using, equivalently, the third integral evaluated in Section 2. When $t_{1}=t_{0}$, only the pure derivative results. The magnitude of this Fourier transform is plotted in Figure 21 on log-linear axes, using the same parameters $\alpha=1, \beta=0.5, t_{0}=5$ and $t_{1}=6$ as in the bipolar function plots shown in Figures 19 and 20. This plot shows a low magnitude value at $\omega=0$, a rise to a peak, and then an exponential falloff with $\omega$. These values at $\omega=0$ and at the peak also depend on the time difference $t_{0}-t_{1}$. 


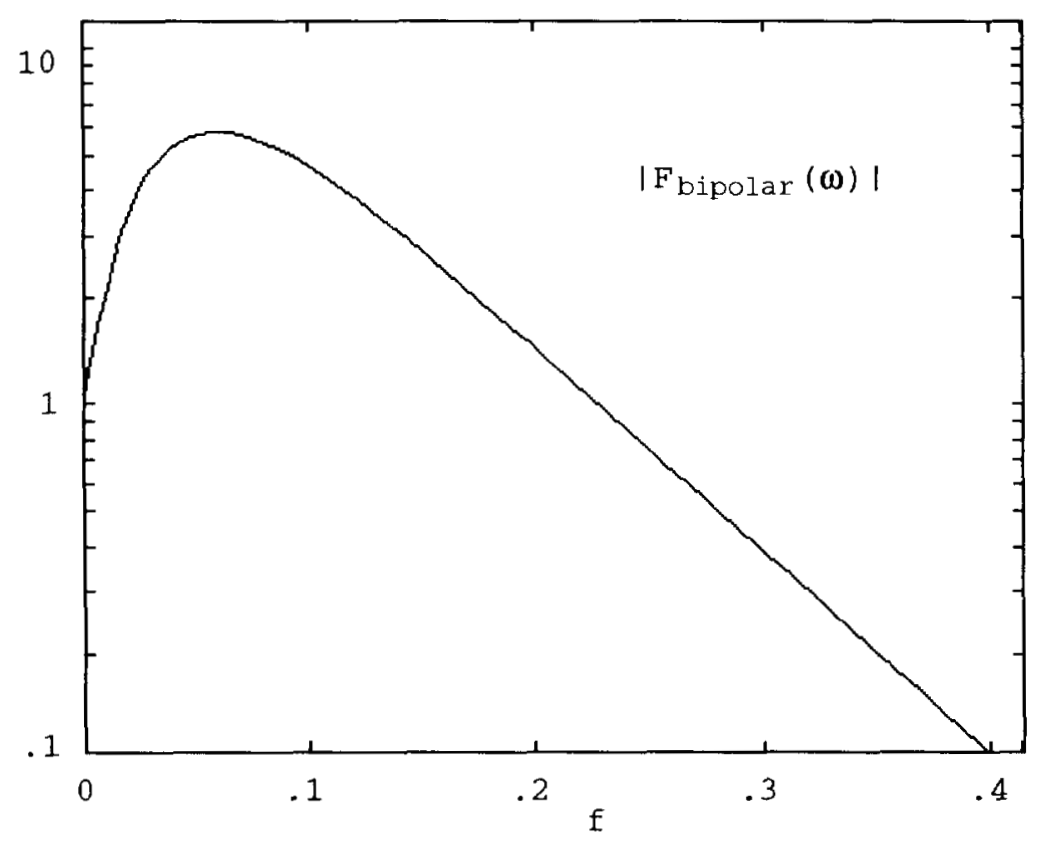

Figure 21. Semi-log plot of the magnitude of the Fourier transform of the bipolar function $\left(t-t_{1}\right) f(t)$ with parameters $\alpha=1, \beta=0.5, t_{0}=5$ and $t_{1}=6$.

\section{Extensions to Boxcar and N Wave Pulse Functions}

We conclude this monograph by describing two other pulse function types that can be derived from the unit step function, and yet have the requisite leading and trailing exponential profiles: these are the boxcar function and the $\mathrm{N}$ wave function. Rounded unit step functions can be modeled as inverse biexponential functions of Section 3 when either the leading or trailing edge becomes horizontal; for this to happen either $\alpha$ or $\beta$ (but not both) become zero. Thus, if we set $\beta=0$ we

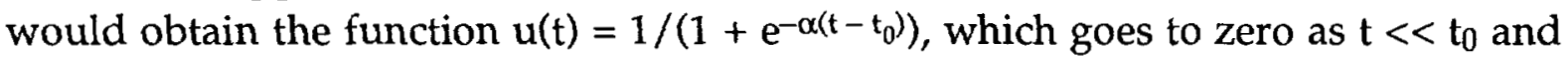
approaches unity for $t>t_{0}$. Also, as $\alpha$ becomes large, the transition in $u(t)$ between 0 and 1 sharpens and becomes discontinuous when $\alpha \rightarrow \infty$. The function formed from the difference of two such $u(t)$ functions (each with a different value of $t_{0}$ ) has a rounded boxcar shape, and can be written

$$
f_{b o x}(t)=\frac{1}{1+e^{-\alpha_{1}\left(t-t_{1}\right)}}-\frac{1}{1+e^{-\alpha_{2}\left(t-t_{2}\right)}} .
$$

This is approximately unity between the times $t_{1}$ and $t_{2}$, and approaches zero on either side of this interval. We plot in Figure 22 an example of this boxcar function with the parameter values $\alpha_{1}=3, t_{1}=0, \alpha_{2}=2$, and $t_{2}=10$. 


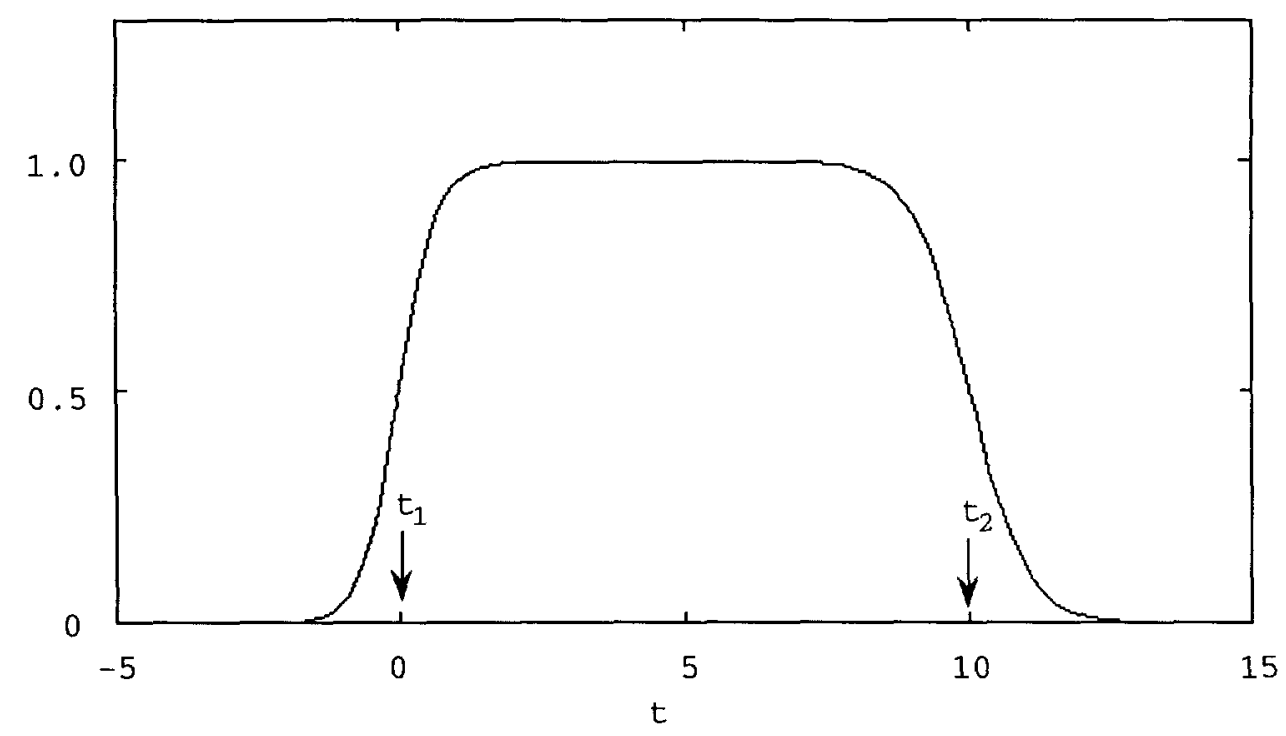

Figure 22. Linear-linear plot of a boxcar function formed as the difference of two rounded step functions with parameters $\alpha_{1}=3, t_{1}=0, \alpha_{2}=2$, and $t_{2}=10$.

The Fourier transform of the boxcar function is easily obtained, because the boxcar function can be written as the sum of two inverse biexponential functions. which is described in detail in Section 4 . The other parameters of the biexponential sum must therefore have the values $\beta_{1}=\beta_{2}=0$ and $\gamma=-1$ in order to produce the boxcar function, and so the Fourier transform of the boxcar follows directly from the formulas derived in Section 4. In Figure 23 we plot the magnitude of the Fourier transform of the boxcar function of Figure 22; the same parameter set is used for both plots. The interference pattern is clearly evident. In the limit as $\alpha_{1}$ and $\alpha_{2}$ both become large, the rounded boxcar shape becomes more like a rectangle, and its Fourier transform approaches the classic $\sin (\omega \Delta t) / \omega$ shape. (This is also apparent from the fact that the Fourier transforms of the two component rounded step functions (which are shown as the two dotted curves in Figure 23) each behave as $1 / \omega$ at low values of $\omega$.)

The $\mathrm{N}$ wave function is obtained from the boxcar function by multiplying the latter by the linear function $\left(t-t_{0}\right)$, where $t_{1}<t_{0}<t_{2}$; this function is therefore

$$
\mathrm{f}_{\text {Nwave }}=\left(\mathrm{t}-\mathrm{t}_{0}\right) \mathrm{f}_{\text {box. }}
$$

An example $\mathrm{N}$ wave function is shown plotted in Figure 24, corresponding to the parameter values $t_{0}=4.5, \alpha_{1}=3, t_{1}=0, \alpha_{2}=4$, and $t_{2}=10$. 


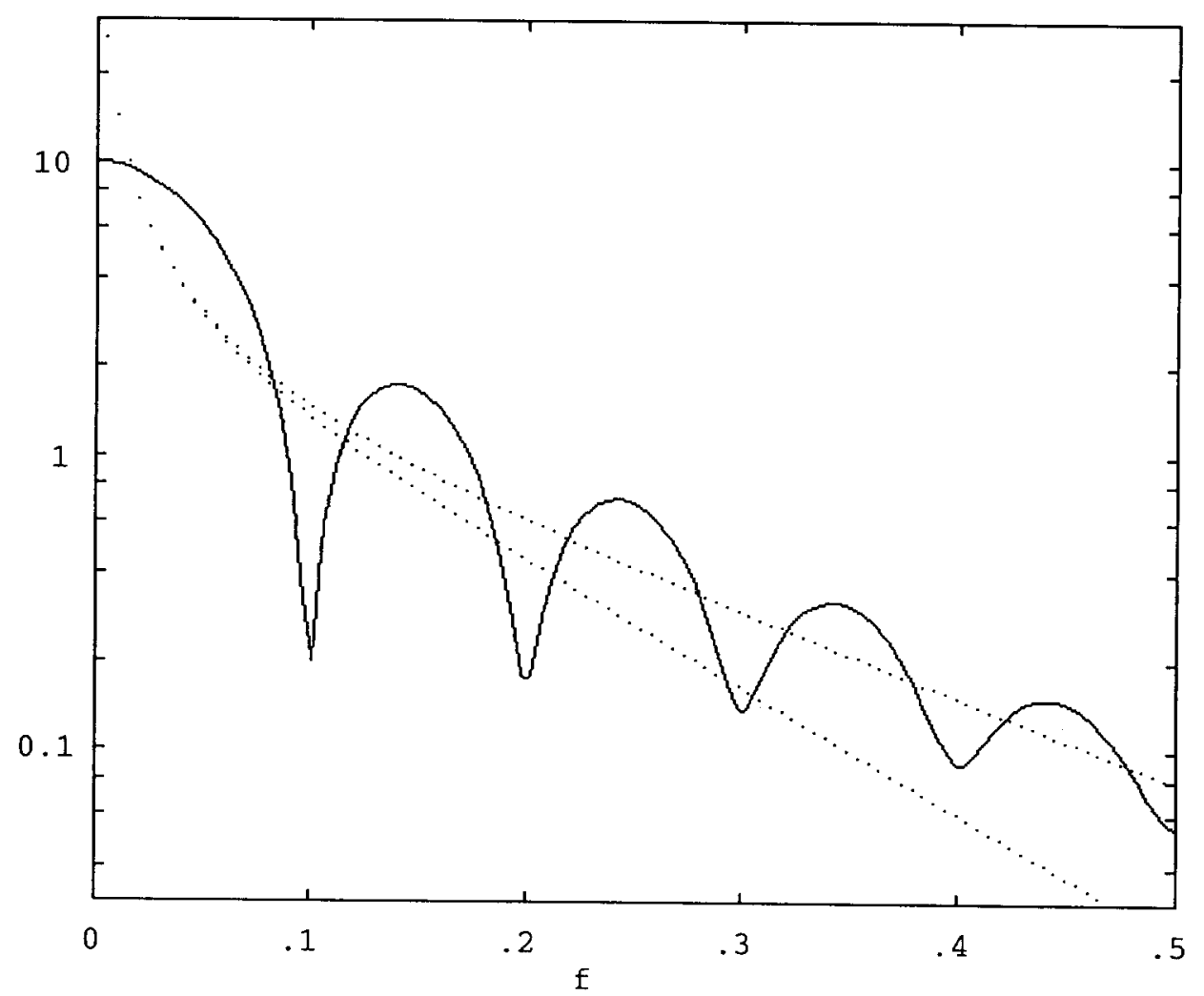

Figure 23. Log-linear plot of the magnitude of the Fourier transform of the boxcar function shown in Figure 22. The dotted curves show the Fourier transform magnitudes of the step function components.

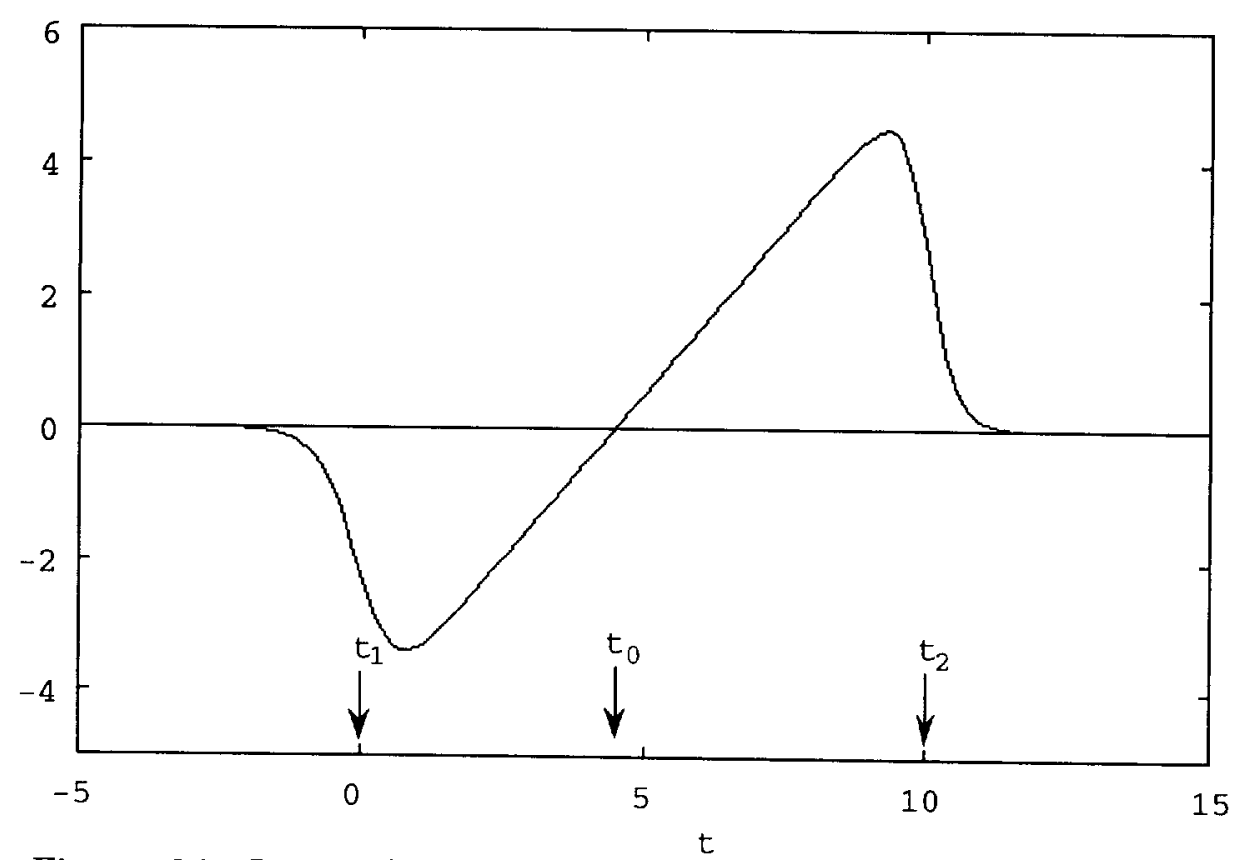

Figure 24. Linear-linear plot of the rounded $\mathrm{N}$ wave function $\left(t-t_{0}\right) f_{\text {box }}(t)$, with parameters $t_{0}=4.5, \alpha_{1}=3, t_{1}=0, \alpha_{2}=4$, and $t_{2}=10$. 
The Fourier transform for the $\mathrm{N}$ wave is also obtained easily, because the $\mathrm{N}$ wave is the difference of two bipolar biexponential functions of the form $\left(t-t_{0}\right) f(t)$ each having by the same linear factor, and such bipolar functions were treated in Section 6. Its Fourier transform is therefore the difference of two transforms each of the form $F_{b i p o l a r}(\omega)$ derived in Section 6. More explicitly, if

$$
\begin{aligned}
f_{\text {Nwave }}(t) & =\frac{\left(t-t_{0}\right)}{1+e^{-\alpha_{1}\left(t-t_{1}\right)}}-\frac{\left(t-t_{0}\right)}{1+e^{-\alpha_{2}\left(t-t_{2}\right)}} \\
& =f_{1 \text { bipolar }}(t)-f_{2 \text { bipolar }}(t)
\end{aligned}
$$

then its Fourier transform is

$$
F_{\text {Nwave }}(\omega)=F_{1 \text { bipolar }}(\omega)-F_{2 \text { bipolar }}(\omega)
$$

where the numerical part of each subscript label indicates a different set of function parameters. We show in Figure 25 a plot of the magnitude of the Fourier transform of the $\mathrm{N}$ wave function displayed in Figure 24; the parameters are the same for both plots. The Fourier transform magnitudes of the two component bipolar functions are indicated by the dotted curves.

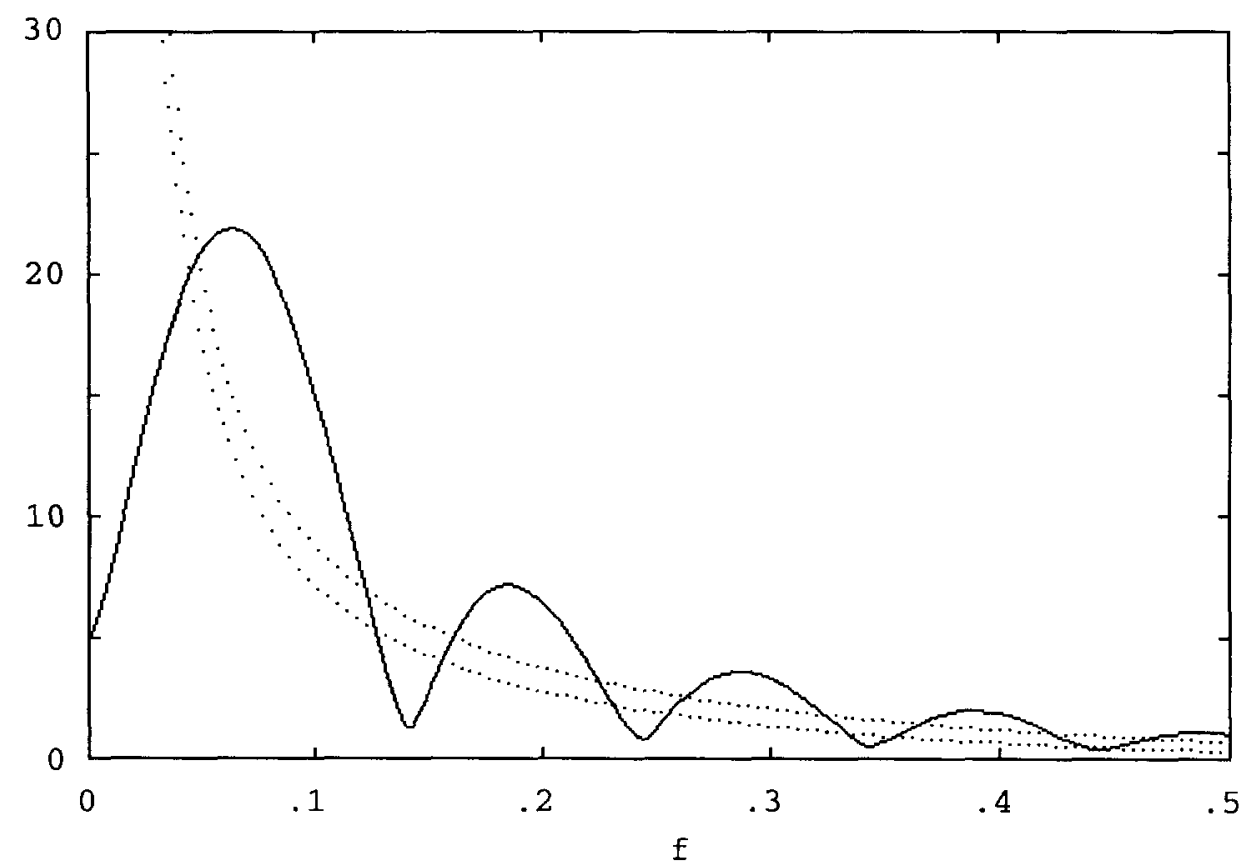

Figure 25. Linear-linear plot of the magnitude of the Fourier transform of the $\mathrm{N}$ wave function shown in Figure 24. The dotted curves show the Fourier transform magnitudes of the bipolar function components. 


\section{Epilogue: Formulas and Algorithms}

Calculating the magnitudes of Fourier transforms presented here does not present difficulties, because the simpler complex multiplication and inversion operations are used, and iterations of these operations may be transferred directly into algorithm form instead of reducing complicated algebraic expressions to simpler forms. Where transforms of sums of functions are involved, the real and imaginary parts are separately summed before taking the magnitude. For reference and convenience the basic calculational procedures are:

Circular functions of complex argument:

$$
\begin{aligned}
& \sin (x+i y)=\sin x \cosh y+i \cos x \sinh y \\
& \cos (x+i y)=\cos x \cosh y-i \sin x \sinh y
\end{aligned}
$$

Inversion: $\quad 1 /(x+i y)=(x-i y) /\left(x^{2}+y^{2}\right)$

Multiplication: $\quad\left(x_{1}+i y_{1}\right)\left(x_{2}+i y_{2}\right)=x_{1} x_{2}-y_{1} y_{2}+i\left(x_{1} y_{2}+x_{2} y_{1}\right)$

Magnitude: $\quad|A+i B|=\left(A^{2}+B^{2}\right)^{1 / 2}$

We next present two examples of Fortran 77 algorithm segments used to calculate the Fourier transforms. The first one, which follows, evaluates the magnitudes of the three "fractal" Fourier transforms worked out in Section 5, which are $F_{1}(\omega), F_{2}(\omega)$ and $F_{3}(\omega)$, for specified $f$. The inputs to this segment are the parameters $\alpha, \beta, \kappa$ and $f$, represented respectively by the variable names alf, bet, ak and $f$. Also, con represents $\kappa \xi=\pi \kappa /(\alpha+\beta)$, and om represents $\omega=2 \pi \mathrm{f}$; the meanings of the other names should be obvious. The magnitude result $|F(\omega)|$ is represented by the variable name ftamp. The algorithm calculation is done for $\kappa=1,2$ or 3 , and in practice $\mathbf{f}$ is stepped as a loop index is incremented, which is not shown. Note that the contribution from the phase factor $\mathrm{e}^{\mathrm{i} \omega \mathrm{t}_{0}}=\cos \left(\omega \mathrm{t}_{0}\right)+\mathrm{i} \sin \left(\omega \mathrm{t}_{0}\right)$ to the real and imaginary parts of the Fourier transforms has been omitted.

The second algorithmic coding segment, which also follows, calculates the magnitude $\left|F_{1}(\omega)+F_{2}(\omega)\right|$ of the Fourier transform of the sum $f_{1}(t)+f_{2}(t)$ of two inverse biexponential functions, as described in Section 4 . The variable names are similar to the ones in the previous example, except that the names of quantities associated with each function have the number 1 or 2 appended, as appropriate. This time the calculation of the phase factors $e^{\mathrm{i} \omega \mathrm{t}_{1}}$ and $\mathrm{e}^{\mathrm{i} \omega \mathrm{t}_{2}}$ is included in order to properly account for interference effects. The inputs to this segment are the parameters $\alpha_{1}, \beta_{1}, t_{1}, \alpha_{2}, \beta_{2}, t_{2}, \gamma$ and $f$, represented respectively by the variable names alf1, bet1, tau1, alf2, bet2, tau2, gam, and $f$. 
Calculation of $\left|F_{1}(\omega)\right|,\left|F_{2}(\omega)\right|$ and $\left|F_{3}(\omega)\right|$, for specified $\omega=2 \pi f$ :

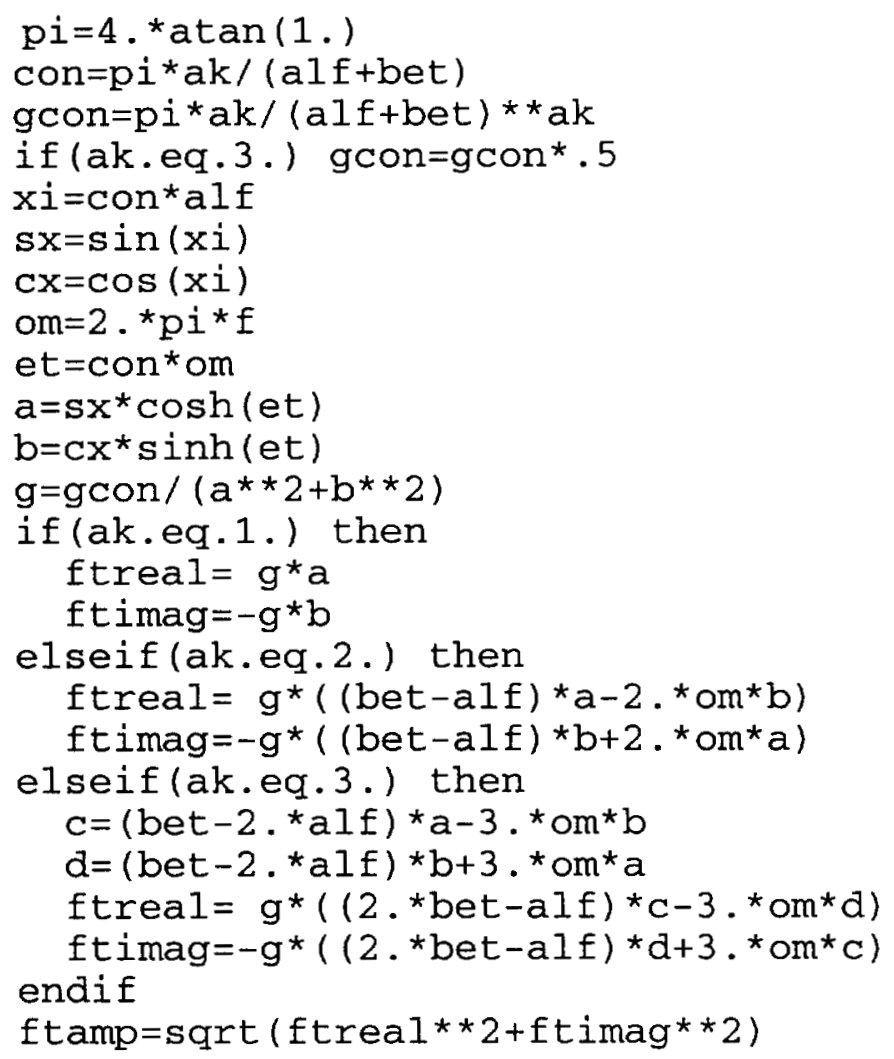

Calculation of $\left|F_{1}(\omega)+F_{2}(\omega)\right|$ for specified $\omega=2 \pi f$ :

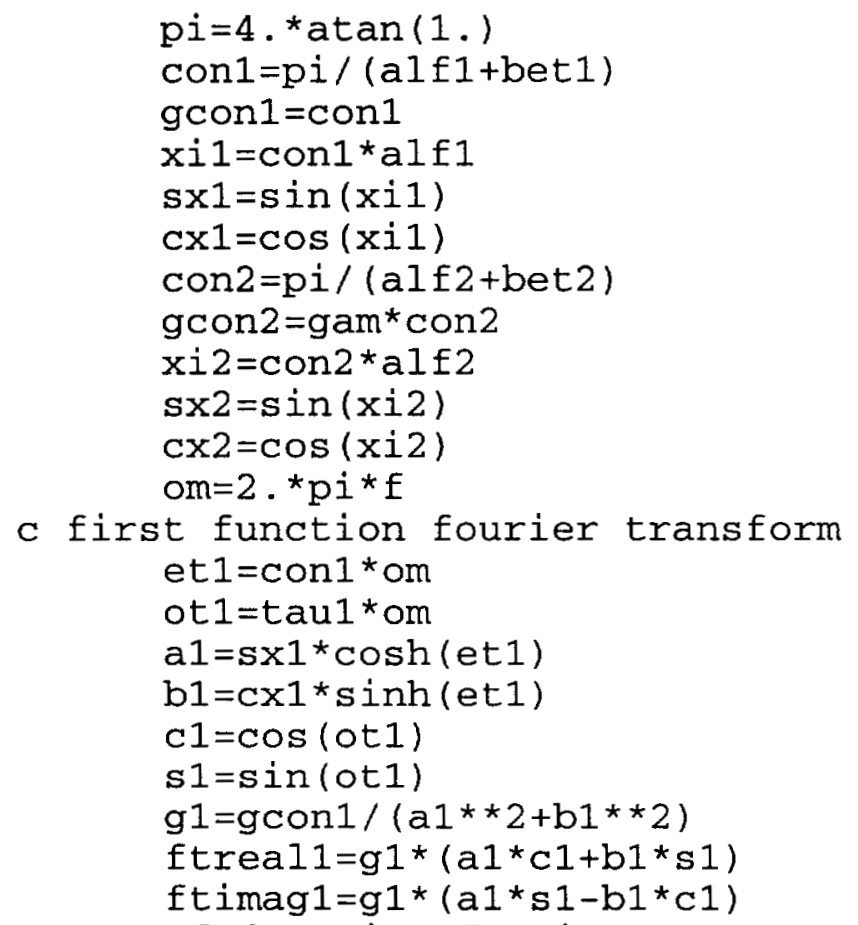




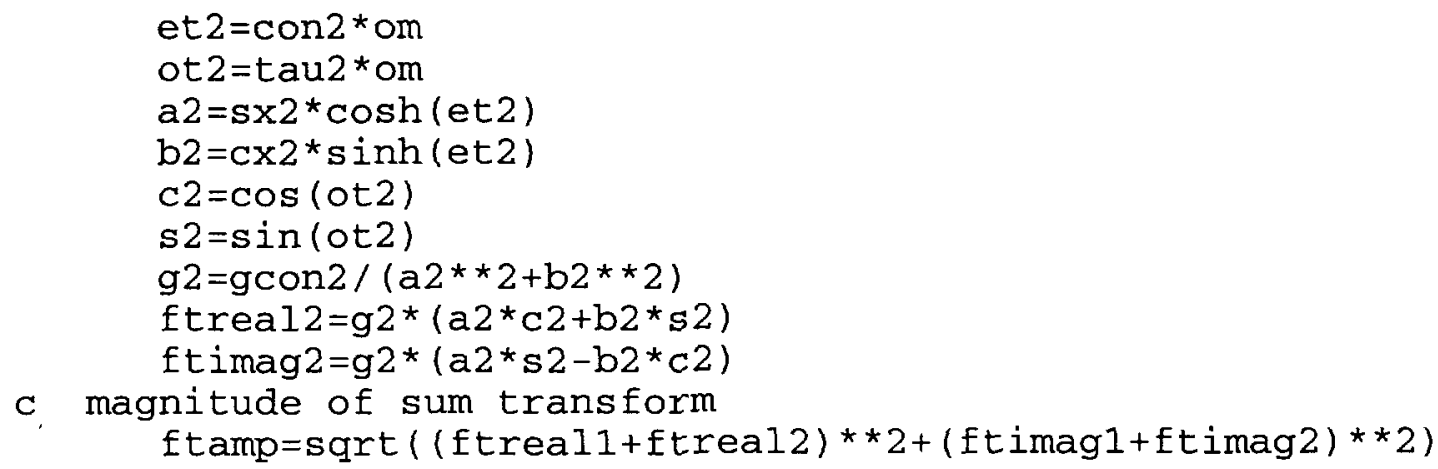

\section{References}

G. B. Whitham, Linear and Nonlinear Waves, John Wiley \& Sons, New York, 1974

A. D. Pierce, Acoustics, McGraw-Hill, New York, 1981

M. J. Lighthill, "Viscosity Effects in Sound Waves of Finite Amplitude", in Surveys in Mechanics, G. K. Batchelor and R. M. Davies, eds., Cambridge University Press, England, 1956

James A. Faulkner, Physics International Co., San Leandro, CA, private communication to D. Lynn Shaeffer, November 1988

Zeev Nehari, Introduction to Complex Analysis, Allyn \& Bacon, Boston, 1961

R. V. Churchill, Introduction to Complex Variables and Applications, McGraw-Hill, New York, 1961 (or any competent complex variables textbook).

D. Lynn Shaeffer, Lawrence Livermore National Laboratory, private communication, December 2001

E. T. Whittaker and G. N. Watson, A Course of Modern Analysis, 4th Edition, Cambridge University Press, England, 1927

T. J. Osler, "Fractional Derivatives and Liebniz Rule", American Mathematical Monthly, Vol. 78, pp. 645 - 649, 1971 (and references cited therein).

Alan Jeffrey, Handbook of Mathematical Formulas and Integrals, Academic Press, New York, 1995

I. S. Gradshteyn and I. M. Ryzhik, Tables of Integrals, Series and Products, 4th Edition, Academic Press, New York, 1965. (In particular, page 292, Section 3.241, integrals 2 and 4 .) 
M. Abramowitz and I. A. Stegun, Handbook of Mathematical Functions with Formulas, Graphs and Mathematical Tables, Dover Publications, New York, 1965.

(The following references describe pulse shape functions and their Fourier transforms similar to some of those discussed in this monograph.)

John Northrop, Ed., Handbook of Nuclear Weapon Effects, Defense Special Weapons Agency*, September 1996 (distribution restricted), Chapter 10.

H. Jerry Longley and Conrad L. Longmire, Development of the CHAP EMP Code, Defense Nuclear Agency* Report DNA 3150T, November 1973 (NTIS accession number AD528424)

William A. Radasky et al, High Altitude Electromagnetic Pulse -- Theory and Calculations, Defense Nuclear Agency* Report DNA-TR-88-123, October 1988

Conrad L. Longmire et al, A Nominal Set of High-Altitude EMP Environments, Mission Research Corp., Santa Barbara CA, Report MRC-R-991R-1, February 1987

* Address: 6801 Telegraph Road, Alexandria VA 
Appendix: Complex Gamma Function Subroutine

For completeness, we include below a listing of the modified Fortran 77 double precision complex gamma function subroutine that was used to help calculate the graphical results presented in Section 5. The original subroutine was downloaded in May 2002 from the following site on the Internet:

http://momonga.t.u-tokyo.ac.jp/ ooura/gamerf .html

The source is evidently the following person:

Takuya OOURA

Research Institute for Mathematical Sciences

Kyoto University

Kyoto 606-01 Japan

The electronic mail address is

or

ooura@kurims.kyoto-u.ac.jp

ooura@mmm.t.u-tokyo.ac.jp

subroutine cdgamma(xreal, ximag, freal, fimag)

real xreal, ximag, freal, fimag

implicit real*8 $(a-h, o-z)$

parameter (

$\& \quad \mathrm{pi}=3.14159265358979324 \mathrm{~d}+00$,

$\& \quad$ pv $=7.31790632447016203 d+00$,

$\& \quad$ pu $=3.48064577727581257 \mathrm{~d}+00$ ，

$\& \quad \mathrm{pr}=3.27673720261526849 \mathrm{~d}-02$ ，

$\& \quad \mathrm{p} 1=1.05400280458730808 \mathrm{~d}+01$,

$\& \quad \mathrm{p} 2=4.73821439163096063 \mathrm{~d}+01$,

$\& \quad \mathrm{p} 3=9.11395751189899762 \mathrm{~d}+01$,

$\& \quad \mathrm{p} 4=6.62756400966213521 \mathrm{~d}+01$,

$\delta \quad \mathrm{p} 5=1.32280130755055088 \mathrm{~d}+01$,

$\& \quad \mathrm{p} 6=2.93729529320536228 \mathrm{~d}-01)$

parameter (

\& $\quad \mathrm{q} 1=9.99999999999975753 \mathrm{~d}-01$,

\& $\quad \mathrm{q} 2=2.00000000000603851 \mathrm{~d}+00$,

\& $\quad \mathrm{q} 3=2.99999999944915534 \mathrm{~d}+00$,

$\& \quad \mathrm{q} 4=4.00000003016801681 \mathrm{~d}+00$,

$\& \quad q 5=4.99999857982434025 d+00$,

$\& \quad q 6=6.00009857740312429 d+00)$

c complex Gamma function in double precision

$$
\begin{gathered}
x r=d b l e(x r e a l) \\
x i=d b l e(x i m a g) \\
\text { if }(x r . l t .0) \text { then } \\
\text { wr }=1-x r \\
\text { wi }=-x i
\end{gathered}
$$




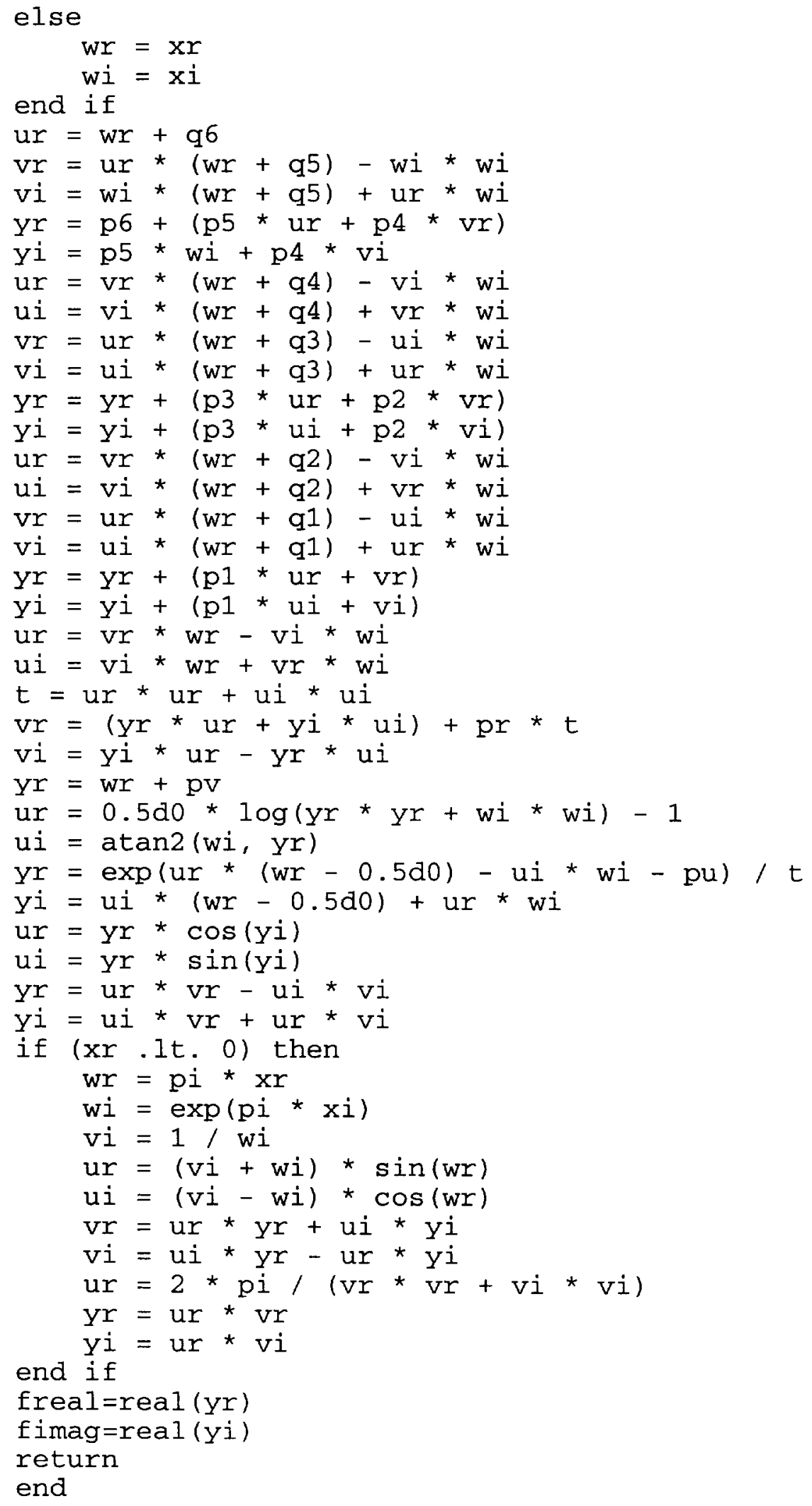

